\title{
Non-vanishing superpotentials in heterotic string theory and discrete torsion
}

Evgeny I. Buchbinder ${ }^{a}$ and Burt A. Ovrut ${ }^{b}$

${ }^{a}$ School of Physics (M013), The University of Western Australia, 35 Stirling Highway, Crawley WA 6009, Australia

${ }^{b}$ Department of Physics and Astronomy, University of Pennsylvania, 209 South 33rd Street, Philadelphia, PA 19104-6395, U.S.A.

E-mail: evgeny.buchbinder@uwa.edu.au, ovrut@elcapitan.hep.upenn.edu

ABstRACT: We study the non-perturbative superpotential in $E_{8} \times E_{8}$ heterotic string theory on a non-simply connected Calabi-Yau manifold $X$, as well as on its simply connected covering space $\tilde{X}$. The superpotential is induced by the string wrapping holomorphic, isolated, genus 0 curves. According to the residue theorem of Beasley and Witten, the non-perturbative superpotential must vanish in a large class of heterotic vacua because the contributions from curves in the same homology class cancel each other. We point out, however, that in certain cases the curves treated in the residue theorem as lying in the same homology class, can actually have different area with respect to the physical Kahler form and can be in different homology classes. In these cases, the residue theorem is not directly applicable and the structure of the superpotential is more subtle. We show, in a specific example, that the superpotential is non-zero both on $\tilde{X}$ and on $X$. On the non-simply connected manifold $X$, we explicitly compute the leading contribution to the superpotential from all holomorphic, isolated, genus 0 curves with minimal area. The reason for the non-vanishing of the superpotental on $X$ is that the second homology class contains a finite part called discrete torsion. As a result, the curves with the same area are distributed among different torsion classes and, hence, do not cancel each other.

KEYwords: Superstring Vacua, Superstrings and Heterotic Strings

ARXIV EPRINT: 1611.01922 


\section{Contents}

1 Introduction 2

2 Non-perturbative superpotentials in heterotic string theory 4

2.1 The general structure of non-perturbative superpotentials 4

2.2 The residue theorem of Beasley-Witten 6

$\begin{array}{lll}2.3 & \text { Applicability of the residue theorem } & 7\end{array}$

2.4 Discrete torsion 8

3 The Schoen manifold and the prepotential 10

$\begin{array}{ll}3.1 \text { The Schoen manifold } & 10\end{array}$

3.2 The ambient space description of the invariant (1,1) classes 13

$\begin{array}{ll}3.3 & \text { The prepotential and Gromov-Witten invariants } \\ \end{array}$

$\begin{array}{ll}3.4 & \text { Explicit construction of the isolated, genus } 0 \text { curves } \\ \end{array}$

4 Non-vanishing of the superpotential on $\tilde{X} \quad 17$

5 The vector bundle $\quad 18$

5.1 Construction of the vector bundle $\tilde{V}$ on $\tilde{X} \quad 19$

5.2 The ambient space description of $\tilde{V} \quad 21$

5.3 Parametrization of the moduli space 24

6 The superpotential on $X$

6.1 Calculation of the Pfaffians 26

6.2 The residue theorem on $\tilde{X} \quad 30$

6.3 The explicit formula for the superpotential on $X$

$\begin{array}{lll}7 & \text { Conclusion and future directions } & 32\end{array}$

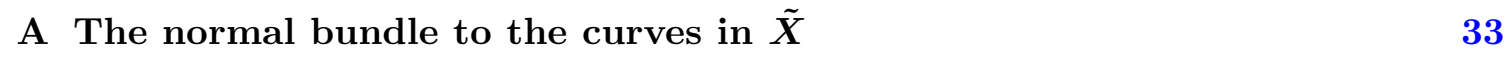

B Extension of $\tilde{W}$ and $\tilde{V}$

B.1 Extensions of $\tilde{W} \quad 34$

$\begin{array}{ll}\text { B.2 Extensions of } \tilde{V} & 35\end{array}$

C Stability of $\tilde{W}$ and $\tilde{V} \quad 36$

$\begin{array}{ll}\text { D Parameterization of the moduli space of } V & 37\end{array}$ 


\section{Introduction}

Compactification of $E_{8} \times E_{8}$ heterotic string theory on smooth Calabi-Yau (CY) threefolds can lead to realistic particle physics models. For example, heterotic M-theory vacua consisting of stable, holomorphic SU(4) vector bundles defined by "extension" over a class of Schoen CY threefolds can produce exactly the spectrum of the minimal supersymmetric standard model (MSSM) with gauged $B-L$ symmetry [1-4]. Similarly, heterotic M-theory compactified on other classes of CY threefolds, such as the tetra-quadric, carrying "monad" vector bundles can lead to the MSSM at low energy, with or without gauged $B-L$ symmetry [5-9]. Although these string vacua realize the correct spectrum and interactions of low energy particle physics, there remains a fundamental problem; that is, that the associated threefolds and vector bundles have moduli that generically have no potential energy. Therefore, the vacuum values of these fields can be dynamically unstable and, even if time-independent, cannot be uniquely specified - thus rendering explicit predictions of the values of supersymmetry breaking and physical parameters impossible. It follows that the stabilization of both geometric and vector bundle moduli is one of the most important problem in heterotic string theory.

A non-vanishing potential energy for the geometric moduli, that is, the complex structure [10] and Kahler moduli, can occur for specific heterotic string vacua due to both perturbative and non-perturbative effects. This leads to partial, and in some toy cases complete, stabilization of these moduli [11]. However, the situation for vector bundle moduli is more difficult. Here, there is no perturbative contribution to their potential energy and one must examine possible non-perturbative effects. A non-perturbative superpotential can, in principal, be generated by string instantons [12-20]. It depends (inversely) exponentially on the Kahler moduli, and also contributes to a potential energy for both complex structure and, importantly, the vector bundle moduli through 1-loop determinants. However, it is difficult to compute these 1-loop quantities. So far, this has only been carried out for specific examples of elliptically fibered CY threefolds with spectral cover vector bundles [21, 22]. It is important, therefore, to generalize these constructions to more realistic vacua, such as those mentioned above. Even then, to find the complete superpotential one has to sum up the contributions from all holomorphic, isolated, genus 0 curves. Beasley and Witten showed that, in a large class of models, these contributions cancel against each other $[23,24]$. Hence, in addition to calculating the instanton generated superpotential for specific curves in more realistic vacua, one must then show that these contributions do not cancel each other; that is, that the Beasley-Witten theorem is not applicable to these theories. In this paper, we take a first step in that direction by explicitly calculating the complete leading order instanton superpotential for a heterotic vacuum consisting of a Schoen [25] threefold geometry and a simple "extension" SU(3) vector bundle - similar, but not identical, to the heterotic standard model in [4]. Although our Schoen threefold is a complete intersection CY manifold (CICY) and the vector bundle descends from a vector bundle on the ambient space - two of the three main conditions required by the Beasley-Witten theorem — we find in this theory that the Beasley-Witten theorem is not applicable and that the superpotential indeed does not vanish. Extending this work to exact heterotic standard model vacua will be carried out elsewhere. 
We start our analysis with a theory on a Schoen threefold $\tilde{X}$ which is a CICY in the ambient space $\mathcal{A}=\mathbb{P}^{1} \times \mathbb{P}^{2} \times \mathbb{P}^{2}$. We will also consider only those vector bundles $\tilde{V}$ on $\tilde{X}$ that descend from a vector bundle $\mathcal{V}$ on $\mathcal{A}$. These vacua satisfy two of the three conditions of the residue theorem of Beasley and Witten and, therefore, one might expect the complete non-perturbative superpotential to vanish. However, we point out that the Beasley-Witten residue theorem additionally assumes that the area of all holomorphic curves on the CICY is computed using the restriction of the Kahler form on the ambient space. Usually, this restriction does give the complete Kahler form on the CY manifold - but there are cases when it does not. These more subtle cases arise when the CICY manifold has more $(1,1)$ classes than does the ambient space. As a result, curves which have the same area with respect to the restriction of the Kahler form of the ambient space, can actually have different area with respect to the true Kahler form on the Calabi-Yau space and, hence, can lie in different homology classes. The Schoen manifold studied in the paper has this property. It has $19(1,1)$ classes whereas the ambient space has only 3 . We show that there are holomorphic, isolated, genus 0 curves in this manifold which are unique in their homology classes despite having the same area with respect to the restriction of the Kahler form of $\mathcal{A}$. Thus, for an arbitrary vector bundle the contributions to the non-perturbative superpotential due to these curves cannot cancel each other because they are weighted with different area. This way, one can get around the Beasley-Witten residue theorem.

Furthermore, our CICY Schoen threefold is chosen to have a freely acting $\mathbb{Z}_{3} \times \mathbb{Z}_{3}$ symmetry group. We then mod our this discrete action, to obtain a non-simply connected Calabi-Yau space with $\pi_{1}=\mathbb{Z}_{3} \times \mathbb{Z}_{3}$. For a toy choice of a vector bundle which descends from the ambient space, the non-perturbative superpotential for all holomorphic, isolated, genus 0 curves with minimal area is computed. For simplicity, we perform our calculations for a fixed complex structure. Hence, the only 1-loop determinant which needs to be computed is the Pfaffian of the Dirac operator on these curves. Since we do not know either the metric or the gauge connection, we use an algebraic method (similar to the one developed in $[21,22])$ to compute the Pfaffians. They turn out to be homogeneous, degree 2 polynomials on the moduli space of vector bundles. We show that the sum of the contributions from these curves is non-zero. Here, the main reason for the non-vanishing of the superpotential is the discrete part of the second homology group, called discrete torsion. Due to torsion, curves which have the same area actually lie in different classes of the second homology group with integer coefficients. These different classes are labeled by the characters of the torsion subgroup - which in the present case is $\mathbb{Z}_{3} \oplus \mathbb{Z}_{3}$. Hence, in this case the non-vanishing of the superpotential can also be attributed to existence of holomorphic, isolated, genus 0 curves which are unique in their integral homology classes.

The paper is organized as follows. In section 2, we start with reviewing the structure of the non-perturbative superpotential in heterotic string theory, mostly following [17]. Then we review the residue theorem of Beasley and Witten, pointing out that it is directly applicable only when the Kahler form on the Calabi-Yau manifold is the restriction of the Kahler form of the ambient space. We also discuss how the structure of the superpotential is modified if the second homology group with integer coefficients contains discrete torsion. Holomorphic, isolated, genus 0 curves which are in the same real homology classes and 
which, hence, have the same area, are distributed among different torsion classes labeled by the characters of the torsion group. These characters arise as extra factors in the superpotential. In section 3, we review some mathematical propeties of the Schoen manifold and of its quotient by $\mathbb{Z}_{3} \times \mathbb{Z}_{3}$. We also review the type II prepotential on the quotient computed in [26-28]. We point out that there are 9 holomorphic, isolated, genus 0 curves of the quotient having the same minimal area, but each lying in its own torsion class. In section 4, we show that the pre-image of these curves on the covering Schoen manifold consists of 81 curves lying in 81 different homology classes. These curves have different area due to the Kahler classes which are non-invariant under $\mathbb{Z}_{3} \oplus \mathbb{Z}_{3}$ and, hence, do not descent to the quotient. In particular, this implies that the superpotential on the Schoen manifold is non-zero. In the remaining part of the paper, we compute the superpotential on the quotient manifold due to the above 9 isolated curves with minimal area. In this paper, we do the calculation for a toy model. More realistic vacua will be discussed elsewhere. In section 5, we construct a toy vector bundle with structure group SU(3) which descends to the quotient manifold. We show that its moduli space is a projective space and find its explicit parametrization. In section 6, we compute the Pfaffian of the Dirac operator on the curves of interest. On each curve, the result is a homogeneous polynomial which we find explicitly up to an overall coefficient. We show that, since all curves are in different integral homology classes, their contributions pick up different torsion factors and, hence, they do not cancel each other. In the Conclusion, we summarize our results and discuss directions for further research. Finally, appendices A, B, C and D are devoted to discussing various technical details.

\section{Non-perturbative superpotentials in heterotic string theory}

\subsection{The general structure of non-perturbative superpotentials}

We consider $E_{8} \times E_{8}$ heterotic string theory compactified to four-dimensions on a CalabiYau threefold X. As was extensively studied in a variety of contexts and papers [12-22], the effective low-energy field theory may, in principle, develop a non-perturbative superpotential for the moduli fields generated by worldsheet/worldvolume instantons. The structure of the instantons is slightly different in the weakly and strongly coupled heterotic string theories. Be that as it may, the superpotential has the same generic form. For concreteness, we will discuss the weakly coupled case where the superpotential is generated by strings wrapping holomorphic, isolated, genus 0 curves in X. ${ }^{1}$ Furthermore, for simplicity, we will restrict our discussion to the "observable" sector; that is, to the superfields associated with the first $E_{8}$ factor of the gauge group. The superpotential is then determined by the classical Euclidean worldsheet action $S_{\mathrm{cl}}$ evaluated on the instanton solution and by the 1-loop determinants of the fluctuations around this solution. Let $C$ be a holomorphic, isolated, genus 0 curve in $\mathrm{X}$. Then the general form of the superpotential induced by a string wrapping $C$ is [17]

$$
W(C)=\exp \left[-\frac{A(C)}{2 \pi \alpha^{\prime}}+i \int_{C} B\right] \frac{\operatorname{Pfaff}\left(\bar{\partial}_{\mathrm{V}_{C}(-1)}\right)}{\left[\operatorname{det}^{\prime}\left(\bar{\partial}_{\mathcal{O}}\right)\right]^{2} \operatorname{det}\left(\bar{\partial}_{\mathrm{NC}}\right)} .
$$

\footnotetext{
${ }^{1}$ Holomorphic, non-isolated and/or higher genus curves contribute to higher order F-term interactions [24].
} 
Let us review various ingredients in this formula. The expression in the exponent is the classical Euclidean action evaluated on $C$. In the first term, $A(C)$, is the area of the curve given by

$$
A(C)=\int_{C} \omega,
$$

where $\omega$ is the Kahler form on $\mathrm{X}$. In the second term, $B$ is the heterotic string $B$-field which, in this expression, can be taken to be a closed 2-form, $d B=0$. Let $\omega_{I}$ be a basis of $(1,1)$-forms on X, $I=1, \ldots, h^{1,1}$. Then we can expand

$$
\omega=\sum_{I=1}^{h^{1,1}} t^{I} \omega_{I}, \quad B=\sum_{I=1}^{h^{1,1}} \phi^{I} \omega_{I} .
$$

Let us define the complexified Kahler moduli

$$
T^{I}=\phi^{I}+i \frac{t^{I}}{2 \pi \alpha^{\prime}} .
$$

Then the exponential prefactor becomes

$$
e^{i \alpha_{I}(C) T^{I}}, \quad \alpha_{I}(C)=\int_{C} \omega_{I} .
$$

By construction $\operatorname{Re}\left(i \alpha_{I}(C) T^{I}\right)<0$.

Note that the exponential factor in (2.1) can also be understood as a map from the curve $C$ to the non-zero complex numbers $\mathbb{C}^{*}$. That is,

$$
C \rightarrow \exp \left[-\frac{A(C)}{2 \pi \alpha}+i \int_{C} B\right] .
$$

Since the value of the integrals depends only on the homology class of the curve, the map is more appropriately expressed as

$$
e^{-S_{\mathrm{cl}}}: H_{2}(\mathrm{X}, \mathbb{Z}) \rightarrow \mathbb{C}^{*} .
$$

However, here there is an important caveat. In eqs. (2.3), (2.7) we are assuming that the moduli space of the $B$-field is connected. As we will discuss below, this is not necessarily the case. Hence, the map (2.7) needs to be refined.

Let us now discuss the 1-loop determinants. The first determinant is the Pfaffian of the Dirac operator which comes from integrating over the right moving fermions in the worldsheet theory. In heterotic compactifications, we have to specify the internal gauge field $A$ on $\mathrm{X}$ which satisfies the Hermitian Yang-Mills equations

$$
F_{m n}=0, \quad F_{\bar{m} \bar{n}}=0, \quad g^{m \bar{n}} F_{m \bar{n}}=0,
$$

where $m$ and $\bar{m}$ are holomorphic and anti-holomorphic indices on $\mathrm{X}$ and $g_{m \bar{n}}$ is the Ricci flat metric on X. According to the theorem of Donaldson-Uhlenbeck-Yau, $A$ is a connection on a holomorphic polystable vector bundle $\mathrm{V}$ on $\mathrm{X}$ whose structure group is a subgroup of $E_{8}$. Then the Pfaffian in (2.1) is the Pfaffian of the Dirac operator depending on the 
connection $A$ restricted to the curve $C$. Since the spin bundle on a genus 0 curve is $\mathcal{O}_{C}(-1)$, we additionally tensor $\mathrm{V}$ with $\mathcal{O}_{C}(-1)$ and denote $\mathrm{V}_{C}(-1)=\left.\mathrm{V}\right|_{C} \otimes \mathcal{O}_{C}(-1) . \operatorname{Pfaff}\left(\bar{\partial}_{\mathrm{V}_{C}(-1)}\right)$ depends on the moduli of the vector bundle $\mathrm{V}$. In principle, it can be explicitly expressed as a function of the gauge connection $A$ using the WZW model [22]. However, since no explicit solutions to the Hermitian Yang-Mills equations on $\mathrm{X}$ are known, it is unclear how to use this in practice. Since right moving worldsheet fermions are Weyl, the $\operatorname{Pfaff}\left(\bar{\partial}_{\mathrm{V}_{C}(-1)}\right)$ is anomalous. However, this anomaly is cancelled by the variation of the $B$-field [22]. As the result, the Pfaffian of the Dirac operator is not a function on the moduli space of $\mathrm{V}$ but, rather, a section of some line bundle. In the denominator in $(2.1), \operatorname{det}\left(\bar{\partial}_{\mathrm{NC}}\right)$ comes from integrating over bosonic fluctuations and is the determinant of the $\bar{\partial}$-operator on the normal bundle to the curve $C$. For an isolated, genus 0 curve, the normal bundle is $N C=\mathcal{O}_{C}(-1) \oplus \mathcal{O}_{C}(-1)$. Hence, $\operatorname{det}\left(\bar{\partial}_{\mathrm{NC}}\right)=\left[\operatorname{det} \bar{\partial}_{\mathcal{O}_{C}(-1)}\right]^{2}$. Finally, $\left[\operatorname{det}^{\prime}\left(\bar{\partial}_{\mathcal{O}}\right)\right]^{2}$ is the $\bar{\partial}$-operator on the trivial line bundle which is a constant.

In general, a given homology class of X contains more than 1 holomorphic, isolated, genus 0 curve. The number of these curves is referred to as to Gromov-Witten invariant. All such curves in the same homology class have the same area, the same classical action and the same exponential prefactor in (2.1). However, the 1-loop determinants, in general, are different. Hence, the contribution to the superpotential from all curves $C_{i}$ in the homology class $[C]$ of the curve $C$ is given by (for simplicity, we remove the constant factor $\left.\left[\operatorname{det}^{\prime}\left(\bar{\partial}_{\mathcal{O}}\right)\right]^{-2}\right)$

$$
W([C])=\exp \left[-\frac{A(C)}{2 \pi \alpha^{\prime}}+i \int_{C} B\right] \sum_{i=1}^{n_{[C]}} \frac{\operatorname{Pfaff}\left(\bar{\partial}_{\mathrm{V}_{C_{i}}(-1)}\right)}{\left[\operatorname{det} \bar{\partial}_{\mathcal{O}_{C_{i}}(-1)}\right]^{2}},
$$

where $n_{[C]}$ is the number of the holomorphic, isolated, genus 0 curves in the homology class $[C]$. To find the complete non-perturbative superpotential $W$, we then have to sum over all homology classes. That is,

$$
W=\sum_{[C] \in H_{2}(\mathrm{X})} W([C]) .
$$

\subsection{The residue theorem of Beasley-Witten}

In [23] (also see earlier papers [29-32]) Beasley and Witten showed that, under some rather general assumptions, the sum (2.9) must vanish for each homology class $[C]$. Here, we review their assumptions since they will be important later in the paper. Let $\tilde{X}$ be a complete intersection Calabi-Yau threefold in the product of projective spaces ${ }^{2} \mathcal{A}=$ $\mathbb{P}^{n_{1}} \times \cdots \times \mathbb{P}^{n_{a}}$. That is, $\tilde{X}$ is given by a set of polynomial equations $p_{1}=0, \ldots, p_{m}=0$ where $\sum_{i=1}^{a} n_{i}-m=3$. Additionally, assume that the Kahler form $\omega_{\tilde{X}}$ descends from the ambient space, that is, $\omega_{\tilde{X}}=\left.\omega_{\mathcal{A}}\right|_{\tilde{X}}$, and that the vector bundle $\tilde{V}$ on $\tilde{X}$ is obtained as a restriction of a vector bundle $\mathcal{V}$ on $\mathcal{A}, \tilde{V}=\left.\mathcal{V}\right|_{\tilde{X}}$. Then, it was shown by Beasley and Witten that if these assumptions are satisfied, the sum (2.9) vanishes for any homology class. This result was proven in [23] and interpreted as a residue theorem.

\footnotetext{
${ }^{2}$ The results of Beasley and Witten are also expected to be valid for complete intersections in toric varieties.
} 
The proof in [23] is based on standard arguments of topological field theory and localization. First, they constructed a topological worldsheet action with target space $\mathcal{A}$ such that there exists a set of supersymmetric vacuum solutions - all with CICY threefold $\tilde{X}$, $\omega_{\tilde{X}}=\left.\omega_{\mathcal{A}}\right|_{\tilde{X}}$ and $\tilde{V}=\left.\mathcal{V}\right|_{\tilde{X}}$. Each such vacuum is associated with a holomorphic curve $C \subset \tilde{X}$. By the standard arguments of topological field theory, the correlation functions in this theory do not depend on the coupling. In one limit of the coupling, the correlators are localized on this set of supersymmetric vacua solutions. This leads to eq. (2.10) for the total superpotential, where one uses the fact that non-isolated and/or higher genus curves only contribute to higher F-term interactions,. In another limit, the same correlators vanish because of unsaturated fermionic zero modes. Hence, $W=0$. Since the exponential factor is different for each homology class, Beasley and Witten concluded that the sum (2.9) vanishes for any homology class. The assumptions of Beasley and Witten are rather general, which means that in a large class of heterotic string models a nonperturbative superpotential cannot be generated. This raises a question of whether moduli in heterotic compactifications can ever be completely stabilized.

The aim of this paper is to present explicit examples where the non-perturbative superpotential is indeed non-zero.

\subsection{Applicability of the residue theorem}

As we have discussed, in the analysis of Beasley and Witten in [23] there is the assumption that $\omega_{\tilde{X}}=\left.\omega_{\mathcal{A}}\right|_{\tilde{X}}$. This assumption is necessary in order for their analysis to be a topological theory on the ambient space with $\tilde{X}$ as a vacuum solution - and, hence, to use their residue theorem. It follows that, in their theorem, the area of all curves in (2.9), (2.10) is measured using the Kahler form $\omega_{\mathcal{A}}$ on $\mathcal{A}$ restricted to $\tilde{X}$. However, there are cases when this restriction is not the same as the physical Kahler form on $\tilde{X}$. Indeed, it is possible that $h^{1,1}(\tilde{X})$ is not the same as $h^{1,1}(\mathcal{A})$ because there can be classes in $\tilde{X}$ which do not come as a restriction of classes from the ambient space. Hence, the residue theorem, strictly speaking, is valid only if $h^{1,1}(\tilde{X})=h^{1,1}(\mathcal{A}) \cdot{ }^{3}$ If $h^{1,1}(\tilde{X})>h^{1,1}(\mathcal{A})$ the residue theorem, though still valid in the topological theory, is not directly applicable to the physical heterotic string theory. In the former case, the area of holomorphic curves is measured using $\left.\omega_{\mathcal{A}}\right|_{\tilde{X}}$. But in the physical theory, it is measured using the actual Kahler form $\omega_{\tilde{X}}$ on $\tilde{X}$. As a result, the curves which have the same area with respect to $\left.\omega_{\mathcal{A}}\right|_{\tilde{X}}$ might have different area with respect to $\omega_{\tilde{X}}$ and, hence, might lie in different homology classes. More precisely, if $h^{1,1}(\tilde{X})>h^{1,1}(\mathcal{A})$ we have

$$
\omega_{\tilde{X}}=\left.\omega_{\mathcal{A}}\right|_{\tilde{X}}+\Delta \omega_{\tilde{X}}
$$

where $\Delta \omega_{\tilde{X}}$ is the contribution to the Kahler form on $\tilde{X}$ from the $(1,1)$ classes which do not come as a restriction of classes from the ambient space. Then the actual area of a curve $C$ is given by

$$
\int_{C} \omega_{\tilde{X}}=\int_{C}\left(\left.\omega_{\mathcal{A}}\right|_{\tilde{X}}+\Delta \omega_{\tilde{X}}\right) \geq\left.\int_{C} \omega_{\mathcal{A}}\right|_{\tilde{X}}
$$

\footnotetext{
${ }^{3}$ Such models were called favorable in [33].
} 
Two curves $C_{1}$ and $C_{2}$ which satisfy

$$
\left.\int_{C_{1}} \omega_{\mathcal{A}}\right|_{\tilde{X}}=\left.\int_{C_{2}} \omega_{\mathcal{A}}\right|_{\tilde{X}}
$$

and appear to lie in the same homology class from the viewpoint of the residue theorem can actually have different area due to different contributions from $\Delta \omega_{\tilde{X}}$ and can lie in different homology classes.

To say it differently, if $h^{1,1}(\tilde{X})>h^{1,1}(\mathcal{A})$ the correlation functions in the topological theory studied in [23] do not coincide with correlation functions in the physical heterotic string theory on $\tilde{X}$. Hence, the cancellation in the residue theorem does not imply an analogous cancellation in the physical theory. However, we can still apply the residue theorem to the physical theory. If in the physical theory we ignore $\Delta \omega_{\tilde{X}}$ and measure the area of all curves using $\left.\omega_{\mathcal{A}}\right|_{\tilde{X}}$ only, then we should have the same cancellation as in the topological theory. Nevertheless, it is important to emphasize that now the cancellation happens among the 1-loop determinants of the curves in different homology classes but having the same area measured by $\left.\omega_{\mathcal{A}}\right|_{\tilde{X}}$. If we restore the actual area using the Kahler form $\omega_{\tilde{X}}$ on $\tilde{X}$, the contributions of these curves might no longer cancel each other because they might lie in different homology classes and have different area. That is, in the physical theory whether or not curves in a given homology class cancel each other cannot be directly deduced from the residue theorem. Below, we will give an example where the cancellation cannot happen simply because each curve is unique in its homology class.

\subsection{Discrete torsion}

Our discussion so far has been missing an important ingredient called discrete torsion. In general, for an arbitrary complex manifold, $\mathrm{X}$, the second homology group with integer coefficients is of the form

$$
H_{2}(\mathrm{X}, \mathbb{Z})=\mathbb{Z}^{k} \oplus \mathrm{G}_{\mathrm{tor}}, \quad k>0,
$$

where $\mathbb{Z}^{k}$ is the free part and $G_{\text {tor }}$ is a finite group called discrete torsion. For example, a discrete torsion factor of $H_{2}(\mathrm{X}, \mathbb{Z})$ can arise when $\mathrm{X}$ is a quotient of another Calabi-Yau manifold by a freely acting discrete isometry group $K-$ as we will discuss below. The existence of the torsion classes affects the $B$-field. Indeed, the $B$-field is an arbitrary closed 2 -form $d B=0$. However, in general, it implies that the field strength $H=d B$ vanishes in $H^{3}(\mathrm{X}, \mathbb{R})$ but not necessarily in $H^{3}(\mathrm{X}, \mathbb{Z})$. In the later case the integral $\int_{C} B$ is not defined because the moduli space of the $B$-field is not connected. From the Universal Coefficient Theorem (see e.g. [34]) it follows that

$$
H_{2}(\mathrm{X}, \mathbb{Z})_{\text {tor }}=H^{3}(\mathrm{X}, \mathbb{Z})_{\text {tor }}
$$

This means that there is one-to-one correspondence between the torsion elements of $H_{2}(\mathrm{X}, \mathbb{Z})$ and the number of the connected components of the moduli space of the $B$ field. These connected components can be labeled by the characters of the discrete group $\mathrm{G}_{\text {tor }}$. Since the $B$-field is not continuous, we have to replace the exponential prefactor 
in $(2.1)$ with a more general map from $H^{2}(\mathrm{X}, \mathbb{Z}) \rightarrow \mathbb{C}^{*}[35]$. While we will continue to denote this map by

$$
e^{-S_{\mathrm{cl}}}: H_{2}(\mathrm{X}, \mathbb{Z}) \rightarrow \mathbb{C}^{*}
$$

it is no longer given by expression (2.6). Specifically, it now depends on the discrete choice of the connected component of the moduli space of the $B$-fields. ${ }^{4}$ Choosing a different connected component gives a different map (2.16). It is possible to describe the map (2.16) more explicitly. Let us define the complexified Kahler form

$$
\omega_{\mathbb{C}}=\sum_{I=1}^{h^{1,1}} T^{I} \omega_{I}=\sum_{I=1}^{h^{1,1}}\left(\phi^{I}+i \frac{t^{I}}{2 \pi \alpha^{\prime}}\right) \omega_{I} .
$$

Then the map (2.6) can be understood as

$$
C \rightarrow \exp \left[i \int_{C} \omega_{\mathbb{C}}\right]=\exp \left[i \omega_{\mathbb{C}} \cdot C\right]
$$

where, in the last step, we view $\omega_{\mathbb{C}}$ as the Poincare dual 4-cycle and $\omega_{\mathbb{C}} \cdot C$ is the intersection of this 4-cycle with the curve $C$. However, $\omega_{\mathbb{C}}$ defined in (2.17) is Poincare dual only to an element of the free part of $H_{4}(\mathrm{X}, \mathbb{Z})$. Clearly, $\omega_{\mathbb{C}}$ should also contain a torsion part. Let $\mathrm{G}_{\text {tor }}$ have $r$ generators $\beta_{1}, \ldots, \beta_{r}$. Then the complete expression for $\omega_{\mathbb{C}}$ is given by

$$
\omega_{\mathbb{C}}=\sum_{I=1}^{h^{1,1}} T^{I} \omega_{I}+\sum_{\alpha=1}^{r} s^{\alpha} \beta_{\alpha},
$$

where, slightly abusing notation, we continue to use the same symbol for the complexified Kahler form including torsion. Since $\beta_{\alpha}$ are torsion elements, it follows that for any $\alpha$ there is an integer $m_{\alpha}$ for which $m_{\alpha} \beta_{\alpha}=0$. Hence, we obtain

$$
\exp \left[i \omega_{\mathbb{C}} \cdot C\right]=e^{i \alpha_{I}(C) T^{I}} \prod_{\alpha=1}^{r} e^{i s^{\alpha} \beta_{\alpha}(C)}, \quad \beta_{\alpha}(C)=\beta_{\alpha} \cdot C .
$$

Since $m_{\alpha} \beta_{\alpha}=0$ and $C$ is arbitrary, it follows that $\chi_{\alpha}=e^{i s^{\alpha}}$ is an $m_{\alpha}$-th root of unity. Hence, $s^{\alpha}$ can take only discrete values parametrizing the connected components of the moduli space of the $B$-field. It also follows that $\chi_{\alpha}$ is a character of $G_{\text {tor }}$. We conclude that mapping (2.6) now generalizes to

$$
C \rightarrow e^{i \alpha_{I}(C) T^{I}} \prod_{\alpha=1}^{r} \chi_{\alpha}^{\beta_{\alpha}(C)} .
$$

The precise values of $\chi_{\alpha}$ depends on the choice of the torsion part of $B$; that is, on the choice of the connected component. Clearly, all curves in the same homology class of $H_{2}(\mathrm{X}, \mathbb{Z})$ have the same value of $\beta_{\alpha}(C)$ and, hence, pick up the same character-dependent factor in (2.21).

\footnotetext{
${ }^{4} \mathrm{~A}$ discrete choice of a vacuum is quite common in heterotic compactifications on non-simply connected Calabi-Yau manifolds. Other discrete choices may involve a choice of the equivariant structure of a vector bundle or a choice of a Wilson line.
} 
Let us now refine eq. (2.9) in the presence of discrete torsion. Let $[C]$ be the homology class of the curve $C$ in $H_{2}(\mathrm{X}, \mathbb{R})=\mathbb{R}^{k}$. As we have just discussed, the curves in $[C]$ do not necessarily lie in the same homology class in $H_{2}(\mathrm{X}, \mathbb{Z})$ because they might belong to different torsion classes. Curves belonging to different torsion classes pick up different characters under the map (2.21). Hence, equation (2.9) is modified to become

$$
W([C])=e^{i \alpha_{I}(C) T^{I}} \sum_{i=1}^{n_{[C]}} \frac{\operatorname{Pfaff}\left(\bar{\partial}_{\mathrm{V}_{C_{i}}(-1)}\right)}{\left[\operatorname{det} \bar{\partial}_{\mathcal{O}_{C_{i}}(-1)}\right]^{2}} \prod_{\alpha=1}^{r} \chi_{\alpha}^{\beta_{\alpha}\left(C_{i}\right)}, \quad[C] \in H_{2}(\mathrm{X}, \mathbb{R}) .
$$

To find the complete non-perturbative superpotential, we have to sum over all homology classes $[C] \in H_{2}(\mathrm{X}, \mathbb{R})$. Later in the paper, we will analyze expression (2.22) for a specific example.

\section{The Schoen manifold and the prepotential}

\subsection{The Schoen manifold}

Having presented the generic discussion above, we now proceed to calculate the nonperturbative superpotential for a specific Calabi-Yau threefold. This manifold, denoted by $X$, is the quotient of a simply connected, complete intersection Calabi-Yau threefold, $\tilde{X}$ — chosen to be a specific Schoen manifold [25] — with respect to its fixed-point free symmetry group $K=\mathbb{Z}_{3} \times \mathbb{Z}_{3}$. This Schoen threefold is defined as follows. We construct $\tilde{X}$ as a compete intersection in the ambient space $\mathcal{A}=\mathbb{P}^{1} \times \mathbb{P}^{2} \times \mathbb{P}^{2}$ with homogeneous coordinates

$$
\left(\left[t_{0}: t_{1}\right],\left[x_{0}: x_{1}: x_{2}\right],\left[y_{0}: y_{1}: y_{2}\right]\right) \in \mathbb{P}^{1} \times \mathbb{P}^{2} \times \mathbb{P}^{2} .
$$

$\tilde{X}$ is then given by a common zero locus of two polynomial equations

$$
\begin{array}{r}
P_{1}\left(t_{0}, t_{1}\right) Q_{1}\left(x_{0}, x_{1}, x_{2}\right)+P_{2}\left(t_{0}, t_{1}\right) Q_{2}\left(x_{0}, x_{1}, x_{2}\right)=0 \\
P_{3}\left(t_{0}, t_{1}\right) Q_{3}\left(y_{0}, y_{1}, y_{2}\right)+P_{4}\left(t_{0}, t_{1}\right) Q_{4}\left(y_{0}, y_{1}, y_{2}\right)=0 .
\end{array}
$$

Here $P_{1}, \ldots, P_{4}$ are homogeneous polynomials of degree 1 and $Q_{1}, \ldots, Q_{4}$ are homogeneous polynomials of degree 3 . For the purposes of this paper, we will restrict $\tilde{X}$ to be given by the following polynomials

$$
\begin{aligned}
& F_{1}=t_{0}\left(x_{0}^{3}+x_{1}^{3}+x_{2}^{3}\right)+t_{1}\left(x_{0} x_{1} x_{2}\right)=0 \\
& F_{2}=\left(\lambda_{1} t_{0}+t_{1}\right)\left(y_{0}^{3}+y_{1}^{3}+y_{2}^{3}\right)+\left(\lambda_{2} t_{0}+\lambda_{3} t_{1}\right)\left(y_{0} y_{1} y_{2}\right)=0 .
\end{aligned}
$$

This manifold is self-mirror with $h^{1,1}=h^{2,1}=19[25,36]$. Note that

$$
h^{1,1}(\tilde{X})>h^{1,1}(\mathcal{A})=3
$$

It follows that on $\tilde{X}$ there are $16(1,1)$ classes which do not arise as the restriction of $(1,1)$ classes from the ambient space. The manifold $\tilde{X}$ defined by (3.3) is invariant under the 
action of the $K=\mathbb{Z}_{3} \times \mathbb{Z}_{3}$ symmetry generated by

$$
\begin{aligned}
& g_{1}:\left\{\begin{array}{l}
{\left[x_{0}: x_{1}: x_{2}\right] \mapsto\left[x_{0}: \zeta x_{1}: \zeta^{2} x_{2}\right]} \\
{\left[t_{0}: t_{1}\right] \mapsto\left[t_{0}: t_{1}\right] \text { (no action) }} \\
{\left[y_{0}: y_{1}: y_{2}\right] \mapsto\left[y_{0}: \zeta y_{1}: \zeta^{2} y_{2}\right]}
\end{array}\right. \\
& g_{2}:\left\{\begin{array}{l}
{\left[x_{0}: x_{1}: x_{2}\right] \mapsto\left[x_{1}: x_{2}: x_{0}\right]} \\
{\left[t_{0}: t_{1}\right] \mapsto\left[t_{0}: t_{1}\right] \text { (no action) }} \\
{\left[y_{0}: y_{1}: y_{2}\right] \mapsto\left[y_{1}: y_{2}: y_{0}\right]}
\end{array}\right.
\end{aligned}
$$

where $\zeta=e^{2 \pi i / 3}$. Note that this discrete symmetry does not act on $\mathbb{P}^{1}$. This action has fixed points on the ambient space $\mathcal{A}$, but not on $\tilde{X}$. Having constructed $\tilde{X}$ with a free $\mathbb{Z}_{3} \times \mathbb{Z}_{3}$ action, we define

$$
X=\tilde{X} /\left(\mathbb{Z}_{3} \times \mathbb{Z}_{3}\right) .
$$

This manifold is also self-mirror with $h^{1,1}=h^{2,1}=3$ [27]. From these Hodge numbers, it follows that $H_{2}(X, \mathbb{R})=\mathbb{R}^{3}$. However, $H_{2}(X, \mathbb{Z})$ is more involved. It was shown in [27] that it contains the discrete torsion subgroup

$$
\mathrm{G}_{\mathrm{tor}}=\mathbb{Z}_{3} \oplus \mathbb{Z}_{3} .
$$

That is, the complete $H_{2}(X, \mathbb{Z})$ is given by

$$
H_{2}(X, \mathbb{Z})=\mathbb{Z}^{3} \oplus \mathbb{Z}_{3} \oplus \mathbb{Z}_{3} .
$$

Let us point out that from the Universal Coefficient Theorem it follows that

$$
H^{2}(X, \mathbb{Z})_{\text {tor }}=H_{1}(X, \mathbb{Z})_{\text {tor }}=\mathbb{Z}_{3} \oplus \mathbb{Z}_{3}
$$

Hence, in the present case, the torsion groups of $H_{2}(X, \mathbb{Z})$ and of $H^{2}(X, \mathbb{Z})$ are the same.

Let us present some mathematical details of $\tilde{X}$ and $X$ following [27]. From eq. (3.3) we see that for fixed $\left[t_{0}: t_{1}\right]$ we have two elliptic curves, one in each $\mathbb{P}^{2}$. Thus, each equation in (3.3) defines a rational elliptic surface $d P_{9} \in \mathbb{P}^{1} \times \mathbb{P}^{2}$ and, hence, $\tilde{X}$ is a double elliptic fibration over $\mathbb{P}^{1}$. The structure of $\tilde{X}$ can be illustrated using the diagram

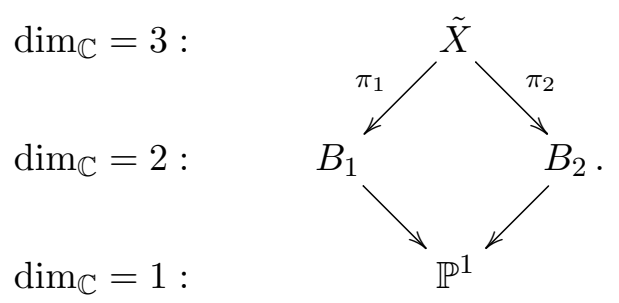

Here $B_{1}$ and $B_{2}$ are the $d P_{9}$ surfaces given by the individual equations in (3.3). The $\mathbb{Z}_{3} \times \mathbb{Z}_{3}$ action descends to $B_{1}$ and $B_{2}$. Since its action is trivial on $\mathbb{P}^{1}$, on each $B_{k}$ for $k=1,2$ the $\mathbb{Z}_{3} \times \mathbb{Z}_{3}$ must act by translation along the fiber by two independent sections of order 3 . To simplify notation, we denote these sections on either $B_{k}$ by the same symbols $\mu$ and $\nu$ 
- unless it is necessary to distinguish them. Additionally, each $B_{k}$ has the zero section $\sigma$. This determines the structure of Kodaira fibers and the Mordell-Weil group to be [37]

$$
\begin{aligned}
\operatorname{sing}\left(B_{1}\right) & =\operatorname{sing}\left(B_{2}\right)=4 I_{3}, \\
M W\left(B_{1}\right) & =M W\left(B_{2}\right)=\mathbb{Z}_{3} \oplus \mathbb{Z}_{3} .
\end{aligned}
$$

The Mordell-Weil group is generated by the zero section $\sigma$ and by the sections $\mu$ and $\nu$ of order 3 . Each $B_{k}$ has $4 I_{3}$ singular fibers, each containing 3 exceptional classes intersecting in a triangle. These classes will be denoted by $\theta_{j i}$, where $j=1, \ldots, 4$ labels the singular fibers and $i=1,2,3$ labels the exceptional classes in each such fiber. As was shown in $[38,39]$, the basis in $H_{2}\left(B_{k}, \mathbb{Z}\right)$ can be chosen to be

$$
H_{2}\left(B_{k}, \mathbb{Z}\right)=\operatorname{span}_{\mathbb{Z}}\left\{\sigma, \mu, \nu, f, \theta_{11}, \theta_{21}, \theta_{31}, \theta_{32}, \theta_{41}, \theta_{42}\right\},
$$

where $f$ is the class of the elliptic fiber. The intersection numbers of these classes can be found in [27]. Out of these basis elements, it is possible to construct divisors which are Poincare dual to $\mathbb{Z}_{3} \times \mathbb{Z}_{3}$ invariant $(1,1)$ classes in $B_{k}$ (here we will use the same notation for divisors and Poincare dual $(1,1)$-forms). The invariant cohomology group of $B_{k}$ is two-dimensional and generated by $[27]$

$$
H^{2}\left(B_{k}, \mathbb{Z}\right)_{K}=\operatorname{span}_{\mathbb{Z}}\{f, t\}
$$

where $t$ is a specific linear combination of the classes in (3.12) given by

$$
t=-3 \sigma-3 f+3 \mu+3 \nu+\theta_{11}+\theta_{21}+2 \theta_{31}+2 \theta_{32}+3 \theta_{41}+\theta_{42} .
$$

The intersection numbers of $f$ and $t$ are [27]

$$
f^{2}=0, \quad f \cdot t=3, \quad t^{2}=1 .
$$

Using the invariant cohomology classes in $B_{k}$, we can now construct divisors in $\tilde{X}$ Poincare dual to the invariant $(1,1)$ classes on $\tilde{X}$. The invariant generators can be defined using the diagram (3.10) and the invariant classes in (3.13):

$$
\begin{aligned}
\phi & =\pi_{1}^{-1}\left(f_{1}\right)=\pi_{2}^{-1}\left(f_{2}\right), \\
\tau_{1} & =\pi_{1}^{-1}\left(t_{1}\right), \quad \tau_{2}=\pi_{2}^{-1}\left(t_{2}\right) .
\end{aligned}
$$

Let us now denote the corresponding Poincare dual (1,1)-forms as $\omega_{\phi}, \omega_{\tau_{1}}, \omega_{\tau_{2}}$. They form a basis of the invariant cohomology group $H^{2}(\tilde{X}, \mathbb{Z})_{K}$, and will descend to the quotient manifold $X$. Their triple intersection numbers can be found using the diagram (3.10) and eq. (3.15) to be

$$
\begin{aligned}
& \int_{\tilde{X}} \omega_{\phi} \wedge \omega_{\tau_{1}} \wedge \omega_{\tau_{2}}=\phi \cdot \tau_{1} \cdot \tau_{2}=9 \\
& \int_{\tilde{X}} \omega_{\tau_{1}} \wedge \omega_{\tau_{1}} \wedge \omega_{\tau_{2}}=\tau_{1} \cdot \tau_{1} \cdot \tau_{2}=3 \\
& \int_{\tilde{X}} \omega_{\tau_{1}} \wedge \omega_{\tau_{2}} \wedge \omega_{\tau_{2}}=\tau_{1} \cdot \tau_{2} \cdot \tau_{2}=3
\end{aligned}
$$


The remaining triple intersection numbers are zero. We can now, somewhat abusing notation, define the form $\omega_{X}$ on $\tilde{X}$ by

$$
\omega_{X}=t^{1} \omega_{\phi}+t^{2} \omega_{\tau_{1}}+t^{3} \omega_{\tau_{2}}, \quad t^{I}>0
$$

which will descend to the non-torsion part of the Kahler form on $X$. Let us emphasize, however, that $\omega_{X}$ is not the same as the Kahler form on $\tilde{X}$. This follows from the fact that on $\tilde{X}$ there are additional classes of $H^{2}(\tilde{X}, \mathbb{Z})$ which are not invariant under $\mathbb{Z}_{3} \times \mathbb{Z}_{3}$. Indeed, as we stated previously, $h^{1,1}(\tilde{X})=19$. This means that there are $16(1,1)$ classes on $\tilde{X}$ in addition to $\omega_{\phi}, \omega_{\tau_{1}}$ and $\omega_{\tau_{2}}$. That is, the complete Kahler form on $\tilde{X}$ is given by

$$
\omega_{\tilde{X}}=\omega_{X}+\Delta \omega_{\tilde{X}}
$$

where $\Delta \omega_{\tilde{X}}$ stands for the contribution from the additional 16 non-invariant classes. Comparing this expression with (2.11), we conclude that

$$
\omega_{X}=\left.\omega_{\mathcal{A}}\right|_{\tilde{X}}
$$

We will give an explanation for this relationship in the following subsection.

\subsection{The ambient space description of the invariant $(1,1)$ classes}

The above description of the invariant $(1,1)$ classes on $\tilde{X}$ is somewhat abstract. Here, we will give a simpler description of $\omega_{\phi}, \omega_{\tau_{1}}, \omega_{\tau_{2}}$ in terms of the forms on the ambient space. Let $\mathcal{J}_{1}, \mathcal{J}_{2}, \mathcal{J}_{3}$ be the Kahler forms on the three projective spaces $\mathbb{P}^{1}, \mathbb{P}^{2}, \mathbb{P}^{2}$ forming the ambient space, normalized as

$$
\int_{\mathbb{P} 1} \mathcal{J}_{1}=1, \quad \int_{\mathbb{P}^{2}} \mathcal{J}_{2} \wedge \mathcal{J}_{2}=1, \quad \int_{\mathbb{P}^{2}} \mathcal{J}_{3} \wedge \mathcal{J}_{3}=1
$$

Since $\mathbb{Z}_{3} \times \mathbb{Z}_{3}$ does not act on $\mathbb{P}^{1}$, the cohomology class of $\mathcal{J}_{1}$ is automatically invariant under $\mathbb{Z}_{3} \times \mathbb{Z}_{3}$. In the cohomology class of $\mathcal{J}_{2}$ (and similarly of $\mathcal{J}_{3}$ ), one can choose a representative to be the Kahler form of the Fubini-Study metric with the Kahler potential

$$
\log \left(\left|x_{0}\right|^{2}+\left|x_{1}\right|^{2}+\left|x_{2}\right|^{2}\right)
$$

which is invariant under $\mathbb{Z}_{3} \times \mathbb{Z}_{3}$. This means that the cohomology classes of $\mathcal{J}_{2}$ and $\mathcal{J}_{3}$ are also invariant classes.

Let us now define the $(1,1)$ classes on $\tilde{X}$ by restriction

$$
J_{1}=\left.\mathcal{J}_{1}\right|_{\tilde{X}}, \quad J_{2}=\left.\mathcal{J}_{2}\right|_{\tilde{X}}, \quad J_{3}=\left.\mathcal{J}_{3}\right|_{\tilde{X}}
$$

By construction, the cohomology classes of $J_{1}, J_{2}, J_{3}$ are invariant classes in $H^{2}(\tilde{X}, \mathbb{Z})$ and, hence, form a basis in $H^{2}(\tilde{X}, \mathbb{Z})_{K}$. The triple intersection numbers of $J_{1}, J_{2}, J_{3}$ can be computed by the standard methods of complete intersection Calabi-Yau manifolds (see e.g. [40] $)^{5}$ with the following result

$$
\begin{aligned}
& \int_{\tilde{X}} J_{1} \wedge J_{2} \wedge J_{3}=9, \\
& \int_{\tilde{X}} J_{2} \wedge J_{2} \wedge J_{3}=3, \quad \int_{\tilde{X}} J_{2} \wedge J_{3} \wedge J_{3}=3,
\end{aligned}
$$

\footnotetext{
${ }^{5}$ One can also compute them by lifting the triple intersection integrals to the ambient space by inserting the delta-function current as in [41-43]. See appendix A in [42] for a similar calculation.
} 
with the remaining ones being zero. Comparing eq. (3.24) with (3.17) we conclude that

$$
\omega_{\phi}=J_{1}, \quad \omega_{\tau_{1}}=J_{2}, \quad \omega_{\tau_{2}}=J_{3} .
$$

That is, the invariant $(1,1)$ classes $\omega_{\phi}, \omega_{\tau_{1}}, \omega_{\tau_{2}}$, which were constructed in the previous subsection in a rather abstract way, are simply the restriction of the Kahler forms on the projective spaces forming the ambient space - thus explaining expression (3.20). Due to the normalization properties (3.21), the forms $J_{1}, J_{2}, J_{3}$ can be viewed as first Chern classes of the following line bundles on $\mathcal{A}$ :

$$
\mathcal{O}_{\mathcal{A}}(1,0,0), \quad \mathcal{O}_{\mathcal{A}}(0,1,0), \quad \mathcal{O}_{\mathcal{A}}(0,0,1) .
$$

This implies the following relations between the line bundles

$$
\mathcal{O}_{\tilde{X}}(\phi)=\left.\mathcal{O}_{\mathcal{A}}(1,0,0)\right|_{\tilde{X}}, \quad \mathcal{O}_{\tilde{X}}\left(\tau_{1}\right)=\left.\mathcal{O}_{\mathcal{A}}(0,1,0)\right|_{\tilde{X}}, \quad \mathcal{O}_{\tilde{X}}\left(\tau_{2}\right)=\left.\mathcal{O}_{\mathcal{A}}(0,0,1)\right|_{\tilde{X}}
$$

These relations will be useful later. For emphasis, we again note note that the Kahler form (3.19) can be written as

$$
\omega_{\tilde{X}}=\left.\omega_{\mathcal{A}}\right|_{\tilde{X}}+\Delta \omega_{\tilde{X}}
$$

We see, therefore, that the Kahler form on $\tilde{X}$ is not simply given by the restriction of the Kahler form from the ambient space but, rather, contains an additional term $\Delta \omega_{\tilde{X}}$.

\subsection{The prepotential and Gromov-Witten invariants}

The number of holomorpic, isolated, genus 0 curves in each homology class of $X$ can be read off from the prepotential in type II string theory. The prepotential on $X$ was computed in [26-28]. In this subsection, we will review the result. Since the $(1,1)$ classes $\left\{\omega_{\phi}, \omega_{\tau_{1}}, \omega_{\tau_{2}}\right\}$ are $\mathbb{Z}_{3} \times \mathbb{Z}_{3}$ invariant on $\tilde{X}$, they descend to cohomology classes on $X$. To simplify our notation, we will label these cohomology classes using the same symbols. Let $\left\{\left[C_{\phi}\right],\left[C_{\tau_{1}}\right],\left[C_{\tau_{2}}\right]\right\}$ be the dual homology classes in the free part of $H_{2}(X, \mathbb{Z})$. If $C_{\phi}, C_{\tau_{1}}$, $C_{\tau_{2}}$ are arbitrary representatives of these classes, then

$$
\int_{C_{\phi}} \omega_{\phi}=1, \quad \int_{C_{\tau_{1}}} \omega_{\tau_{1}}=1, \quad \int_{C_{\tau_{2}}} \omega_{\tau_{2}}=1,
$$

with the other integrals being zero. Let us define

$$
\begin{aligned}
& p=e^{-S_{\mathrm{cl}}}\left(\left[C_{\phi}\right]\right)=\exp \left[\int_{C_{\phi}}\left(-\frac{\omega_{X}}{2 \pi \alpha^{\prime}}+i B\right)\right]=e^{i T^{1}}, \\
& q=e^{-S_{\mathrm{cl}}}\left(\left[C_{\tau_{1}}\right]\right)=\exp \left[\int_{C_{\tau_{1}}}\left(-\frac{\omega_{X}}{2 \pi \alpha^{\prime}}+i B\right)\right]=e^{i T^{2}}, \\
& r=e^{-S_{\mathrm{cl}}}\left(\left[C_{\tau_{2}}\right]\right)=\exp \left[\int_{C_{\tau_{2}}}\left(-\frac{\omega_{X}}{2 \pi \alpha^{\prime}}+i B\right)\right]=e^{i T^{3}} .
\end{aligned}
$$

Since $H_{2}(X, \mathbb{Z})$ contains torsion classes, we also have to introduce the image under the map $e^{-S_{\mathrm{cl}}}$ of the $\mathbb{Z}_{3} \times \mathbb{Z}_{3}$ torsion generators - which we denote by $b_{1}$ and $b_{2}$ respectively and satisfy $b_{1}^{3}=b_{2}^{3}=1$. Let $[C]$ be a homology class of the form

$$
[C]=\left(n_{1}, n_{2}, n_{3}, m_{1}, m_{2}\right) \in H_{2}(X, \mathbb{Z})=\mathbb{Z}^{3} \oplus \mathbb{Z}_{3} \oplus \mathbb{Z}_{3}, \quad m_{1}, m_{2}=0,1,2 .
$$


Then, as shown in (2.21), the image of this class under $e^{-S_{\mathrm{cl}}}$ is given by

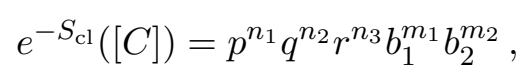

where $b_{1}^{m_{1}} b_{2}^{m_{2}}$ is a $\mathbb{Z}_{3} \times \mathbb{Z}_{3}$ character. The prepotential in type II string theory is defined by the expression

$$
\mathcal{F}_{X}=\sum_{[C] \in H_{2}(X, \mathbb{Z})} n_{[C]} \operatorname{Li}_{3}\left(e^{-S_{\mathrm{cl}}}([C])\right)=\sum_{[C] \in H_{2}(X, \mathbb{Z})} n_{[C]} \operatorname{Li}_{3}\left(p^{n_{1}} q^{n_{2}} r^{n_{3}} b_{1}^{m_{1}} b_{2}^{m_{2}}\right) .
$$

Here the sum is over all holomorphic, isolated, genus 0 curves and the polylogarithm $\mathrm{Li}_{3}$ takes proper care of multiple wrappings. If we know $\mathcal{F}_{X}$, we can expand it in powers of $p, q, r, b_{1}, b_{2}$ and read off the Gromov-Witten invariants $n_{[C]}$. The prepotential $\mathcal{F}_{X}$ for the quotient Calabi-Yau manifold $X$ in (3.6) was computed in [26-28]. Here, we present the result to low orders in $p, q, r$. It is given by

$$
\mathcal{F}_{X}=p\left(1+b_{1}+b_{1}^{2}\right)\left(1+b_{2}+b_{2}^{2}\right) \mathcal{P}(q)^{4} \mathcal{P}(r)^{4}+\mathcal{O}\left(p^{2}\right)
$$

where the polynomial $\mathcal{P}(q)$ is of the form

$$
\mathcal{P}(q)=1+\mathcal{O}(q)
$$

Let us discuss some simple consequences of eqs. (3.34), (3.35). It follows that there are no terms $\sim p^{0}$. In other words, there are no isolated, genus 0 curves in the homology classes $\left(0, n_{2}, n_{3}, m_{1}, m_{2}\right)$. However, there are terms in $\mathcal{F}_{X}$ that are $\sim p^{1}$. Hence, there are isolated, genus zero 0 curves in the $\left(1, n_{2}, n_{3}, m_{1}, m_{2}\right)$ homology classes. It follows from (3.30) that the contribution of these classes to $e^{-S_{\mathrm{cl}}}$ and, hence, the superpotential is proportional to $e^{i T^{1}+i n_{2} T^{2}+i n_{3} T^{3}}$. This means that the leading contribution to the superpotential is $\sim e^{i T^{1}}$; all terms with $n_{2}>0$ and/or $n_{3}>0$ being exponentially suppressed. Similarly, the contribution to the superpotential of any class with $n_{1}>1$ is also suppressed relative to $e^{i T^{1}}$. Therefore, since we are interested in computing the superpotential, we will focus on the homology classes of the form $\left(1,0,0, m_{1}, m_{2}\right)$. The number of isolated, genus 0 curves in each such class can be read off from the most leading term in (3.34). This is given by

$$
\mathcal{F}_{X} \sim p\left(1+b_{1}+b_{1}^{2}\right)\left(1+b_{2}+b_{2}^{2}\right)
$$

It follows that in each torsion class there is precisely 1 curve. That is,

$$
n_{[C]}=1 \quad \text { for each class } \quad[C]=\left(1,0,0, m_{1}, m_{2}\right), m_{1}, m_{2}=0,1,2 .
$$

The main aim of the rest of this paper will be to compute the non-perturbative superpotential (2.22) summed over these 9 isolated, genus 0 curves. All of them are in the same homology class in $H_{2}(X, \mathbb{R})$ and, hence, have the same area with respect to the Kahler form on $X$. However, they are distributed in 9 different homology classes once we take discrete torsion into account. 


\subsection{Explicit construction of the isolated, genus 0 curves}

It is possible to explicitly visualize these 9 curves as follows. We see from eq. (3.30) that, ignoring torsion, they are in the same homology class $\left[C_{\phi}\right]$ which is dual to the class of the $(1,1)$-form $\omega_{\phi}$. Let us lift these curves from $X$ to $\tilde{X}$. Since on $\tilde{X}$ we have $\omega_{\phi}=J_{1}=\left.\mathcal{J}_{1}\right|_{\tilde{X}}$, where $\mathcal{J}_{1}$ is the Kahler form on $\mathbb{P}^{1} \subset \mathcal{A}$, the pre-image of these 9 curves gives 81 holomorphic, genus 0 curves on $\tilde{X}$ which can be parametrized by $\left[t_{0}: t_{1}\right]$. Hence, we can visualize these curves by demanding that eqs. (3.3) are solved for arbitrary $\left[t_{0}: t_{1}\right]$. This is equivalent to solving the system of equations

$$
x_{0} x_{1} x_{2}=0, \quad x_{0}^{3}+x_{1}^{3}+x_{2}^{3}=0, \quad y_{0} y_{1} y_{2}=0, \quad y_{0}^{3}+y_{1}^{3}+y_{2}^{3}=0
$$

on $\mathbb{P}^{2} \times \mathbb{P}^{2}$. It is easy to see that this system is solved by $9 \times 9=81$ distinct points on $\mathbb{P}^{2} \times \mathbb{P}^{2}$. Since the solutions of (3.38) are distinct points, all the corresponding curves in $\tilde{X}$ are isolated. In appendix A we compute the normal bundle for each of these curves and check that it is indeed $\mathcal{O}(-1) \oplus \mathcal{O}(-1)$. Due to the $\mathbb{Z}_{3} \times \mathbb{Z}_{3}$ symmetry, the above 81 curves split into 9 orbits under the action of $\mathbb{Z}_{3} \times \mathbb{Z}_{3}$ - each orbit containing 9 curves. The curves in the same orbit are obtained from each other by the action of the $\mathbb{Z}_{3} \times \mathbb{Z}_{3}$ group. When we descend to the quotient manifold $X$, all curves in one orbit yield the same curve in $X$. Hence, we obtain 9 isolated, genus 0 curves in $X$ which are precisely the curves discussed at the end of the previous subsection. It follows from the prepotential (3.36) they are in the same homology class in $H_{2}(X, \mathbb{R})$ but in 9 different homology classes in $H_{2}(X, \mathbb{Z})$.

We now present 9 curves in $\tilde{X}$ which do not lie in the $\mathbb{Z}_{3} \times \mathbb{Z}_{3}$ orbits of each other and which, therefore, descend to 9 distinct curves in $X$. To accomplish this, let us write the generators $g_{1}$ and $g_{2}$ in (3.5) in the matrix form

$$
g_{1}=\left(\begin{array}{ccc}
1 & 0 & 0 \\
0 & \xi & 0 \\
0 & 0 & \xi^{2}
\end{array}\right), \quad g_{2}=\left(\begin{array}{lll}
0 & 1 & 0 \\
0 & 0 & 1 \\
1 & 0 & 0
\end{array}\right) .
$$

Since $\mathbb{Z}_{3} \times \mathbb{Z}_{3}$ acts simultaneously on both $\mathbb{P}^{2}$ 's, it is convenient to combine $\left[x_{0}: x_{1}: x_{2}\right]$ and $\left[y_{0}: y_{1}: y_{2}\right]$ into a 6 -vector $\left(x_{0}, x_{1}, x_{2}, y_{0}, y_{1}, y_{2}\right)^{T}$. In this basis, the generators of $\mathbb{Z}_{3} \times \mathbb{Z}_{3}$ are

$$
K_{1}=\left(\begin{array}{cc}
g_{1} & 0 \\
0 & g_{1}
\end{array}\right), \quad K_{2}=\left(\begin{array}{cc}
g_{2} & 0 \\
0 & g_{2}
\end{array}\right)
$$

Now choose one arbitrary solution of (3.38). For example, pick

$$
s_{1}=(1,-1,0,1,-1,0)^{T},
$$

where the symbol " $\mathrm{T}$ " means think of this as a column vector. It corresponds to the curve

$$
C_{1}=\mathbb{P}^{1} \times s_{1}=\left[t_{0}: t_{1}\right] \times[1:-1: 0] \times[1:-1: 0] \subset \tilde{X} \subset \mathbb{P}^{1} \times \mathbb{P}^{2} \times \mathbb{P}^{2} .
$$


Let us now construct the remaining 8 curves $C_{i}=\mathbb{P}^{1} \times s_{i}, i=2, \ldots, 9$ by acting on $s_{1}$ as follows:

$$
\begin{aligned}
& s_{2}=\left(\begin{array}{cc}
g_{1} & 0 \\
0 & 1
\end{array}\right) s_{1}, \quad s_{3}=\left(\begin{array}{cc}
1 & 0 \\
0 & g_{1}
\end{array}\right) s_{1}, \quad s_{4}=\left(\begin{array}{cc}
g_{2} & 0 \\
0 & 1
\end{array}\right) s_{1}, \quad s_{5}=\left(\begin{array}{cc}
1 & 0 \\
0 & g_{2}
\end{array}\right) s_{1}, \\
& s_{6}=\left(\begin{array}{cc}
g_{1} g_{2} & 0 \\
0 & 1
\end{array}\right) s_{1}, \quad s_{7}=\left(\begin{array}{cc}
1 & 0 \\
0 & g_{1} g_{2}
\end{array}\right) s_{1}, \quad s_{8}=\left(\begin{array}{cc}
g_{1} & 0 \\
0 & g_{2}
\end{array}\right) s_{1}, \quad s_{9}=\left(\begin{array}{cc}
g_{2} & 0 \\
0 & g_{1}
\end{array}\right) s_{1} \text {. }
\end{aligned}
$$

One can check that these curves solve eqs. (3.38) and cannot be obtained from each other by the action of $\mathbb{Z}_{3} \times \mathbb{Z}_{3}$.

\section{Non-vanishing of the superpotential on $\tilde{X}$}

Let us now compactify the $E_{8} \times E_{8}$ heterotic string on the manifold $\tilde{X}$, and consider the nonperturbative superpotential generated by the 81 isolated, genus 0 curves discussed above. To describe the complete string vacuum, one must also introduce a specific holomorphic vector bundle - which we will do in the next section. Although we will compute the superpotential for this specific bundle, the results of the present section are valid for any vector bundle. Since the curves specified by the solutions of (3.38) are all parameterized by $\left[t_{0}: t_{1}\right]$, it is tempting to conclude that they are in the same homology class dual to the $(1,1)$ class $\omega_{\phi}=\left.\mathcal{J}_{1}\right|_{\tilde{X}}$. However, we will see that this is not the case. In fact, we will show that these curves lie in 81 different homology classes.

First consider the twofold $B_{1} \simeq d P_{9} \subset \mathbb{P} \times \mathbb{P}^{2}$ defined by

$$
F_{1}=t_{0}\left(x_{0}^{3}+x_{1}^{3}+x_{2}^{3}\right)+t_{1}\left(x_{0} x_{1} x_{2}\right)=0 .
$$

Let us now examine the genus 0 curves parametrized by $\left[t_{0}: t_{1}\right]$. They are specified by the 9 solutions of

$$
x_{0} x_{1} x_{2}=0, \quad x_{0}^{3}+x_{1}^{3}+x_{2}^{3}=0 .
$$

Each solution is a distinct section of the elliptically fibered surface $B_{1} \simeq d P_{9}$. We denote these sections by $\sigma_{i}, i=1, \ldots, 9$. Since the order of the Mordell-Weil group $M W\left(B_{1}\right)=Z_{3} \oplus Z_{3}$ is 9 , these sections are in one-to-one correspondence with the elements of $M W\left(B_{1}\right)$ [27]. On the other hand, it was shown in [38] that on a $d P_{9}$ twofold distinct elements of the Mordell-Weil group are all non-homologous to each other. It follows that the 9 solutions to (4.2) fall into 9 different homology classes. The same is true on $B_{2} \simeq d P_{9}$ defined by

$$
F_{2}=\left(\lambda_{1} t_{0}+t_{1}\right)\left(y_{0}^{3}+y_{1}^{3}+y_{2}^{3}\right)+\left(\lambda_{2} t_{0}+\lambda_{3} t_{1}\right)\left(y_{0} y_{1} y_{2}\right)=0
$$

for the 9 genus 0 curves specified by the solutions of

$$
y_{0} y_{1} y_{2}=0, \quad y_{0}^{3}+y_{1}^{3}+y_{2}^{3}=0 .
$$

For reasons of simplicity, we also denote these sections by $\sigma_{i}, i=1, \ldots, 9$. 
We now extend this result to $\tilde{X}$. To start with, note that there is a natural map

$$
H_{2}(\tilde{X}, \mathbb{Z}) \rightarrow H_{2}\left(B_{1}, \mathbb{Z}\right) \times H_{2}\left(B_{2}, \mathbb{Z}\right) .
$$

Let us define

$$
\sigma_{i} \underline{\times} \sigma_{j}=\left(\sigma_{i} \times \sigma_{j}\right) \cap \tilde{X} \in H_{2}(\tilde{X}, \mathbb{Z}), \quad \sigma_{i} \in M W\left(B_{1}\right), \quad \sigma_{j} \in M W\left(B_{2}\right) .
$$

This provides a more abstract way to visualize the 81 curves solving eqs. (3.38). Then the map (4.5) acts on the above described solutions as

$$
\sigma_{i} \underline{\times} \sigma_{j} \rightarrow \sigma_{i} \times \sigma_{j} \in H_{2}\left(B_{1}, \mathbb{Z}\right) \times H_{2}\left(B_{2}, \mathbb{Z}\right) .
$$

We note that the 81 elements $\sigma_{i} \times \sigma_{j}$, all being distinct, are in one-to-one correspondence with elements of the Mordell-Weil group $M W\left(B_{1} \times B_{2}\right)$ of $B_{1} \times B_{2}$ - which is given by $M W\left(B_{1}\right) \oplus M W\left(B_{2}\right)$ and is of order 81 as well. Therefore, we have a map between the set $\left\{\sigma_{i} \times \sigma_{j}\right\}$ and the elements of $M W\left(B_{1}\right) \oplus M W\left(B_{2}\right)$. By construction, it is a surjective linear map between two finite sets consisting of 81 elements each. Hence, it is one-to-one. Since all distinct elements of $M W\left(B_{1}\right) \oplus M W\left(B_{2}\right)$ are non-homologous to each other, it follows that all 81 curves obtained in (3.38) lie in 81 different homology classes. In particular, it follows that each of these 81 homology classes has precisely 1 isolated, genus 0 curve. Hence, as long as the Pfaffian of the Dirac operator of at least one of these curves is not identically zero - which is expected to be true for a generic vector bundle - the nonperturbative superpotential in this theory is non-zero. We will show this explicitly for a specific holomorphic vector bundle in the remainder of this paper.

To finish this section, let us point out that any of the 81 homology classes discussed above are dual to a $(1,1)$ class of the form $\left.\mathcal{J}_{1}\right|_{\tilde{X}}+\Delta \omega_{\tilde{X}} \cdot{ }^{6}$ Here, it suffices to recall that $\Delta \omega_{\tilde{X}}$ stands for the classes on $\tilde{X}$ which cannot be obtained as a restriction from the ambient space. All of these 81 curves have equal area with respect to $\left.\mathcal{J}_{1}\right|_{\tilde{X}}$ and $\left.\omega_{\mathcal{A}}\right|_{\tilde{X}}$, but have different areas with the respect to the actual Kahler form $\omega_{\tilde{X}}$. Hence, we have an explicit realization of the situation described in subsection 2.3. As was discussed, this violates one of the assumptions of the Beasley-Witten residue theorem and, hence, one can expect a non-vanishing instanton superpotential. Finally, note that since $\Delta \omega_{\tilde{X}}$ contains only non-invariant classes under $\mathbb{Z}_{3} \times \mathbb{Z}_{3}$ which vanish on the quotient manifold $X$, the area of the images of the 9 curves in eqs. (3.41), (3.42), (3.43) in $X$ is the same with respect to the Kahler form $\omega_{X}$ on $X$. That is, although the original 81 curves were in different homology classes on the covering manifold, their images are in the same homology class on the quotient manifold modulo torsion.

\section{The vector bundle}

The rest of the paper will be devoted to an explicit computation of the superpotential in a concrete example of a theory on $X$. Specifically, we will consider a toy model where

\footnotetext{
${ }^{6}$ Explicit calculation of $\Delta \omega_{\tilde{X}}$ for each of the 81 curves is a tedious, complicated task which is unnecessary for the purposes of the paper.
} 
the holomorphic vector bundle $V$ is taken to have structure group $\mathrm{SU}(3)$. A more phenomenologically realistic class of bundles will be studied elsewhere. Since $X$ is a quotient manifold, it is easiest to first construct a vector bundle $\tilde{V}$ on the covering space $\tilde{X}$ which is equivariant under the action of $K=\mathbb{Z}_{3} \times \mathbb{Z}_{3}$. The moduli space of an equivariant vector bundle $\tilde{V}$ consists of connected components labeled by the characters of $K$. The vector bundle $V$ on the quotient space $X$ is then defined as

$$
V=\tilde{V} /\left(\mathbb{Z}_{3} \times \mathbb{Z}_{3}\right),
$$

with the moduli space of $V$ consisting of one of the connected components. The choice of the connected component is referred to as to the choice of the equivariant structure. Different choices of equivariant structure give different vector bundles $V$ in (5.1).

First, we will discuss a construction of $\tilde{V}$ in terms of the data on $\tilde{X}$. We then will express the same vector bundle as the restriction of a vector bundle $\tilde{\mathcal{V}}$ on the ambient space. The second description is more explicit and will be used in computing the superpotential in the next section.

\subsection{Construction of the vector bundle $\tilde{V}$ on $\tilde{X}$}

We will construct $\tilde{V}$ by specifying the line bundles $L_{1}, L_{2}, L_{3}$ on $\tilde{X}$ satisfying the property

$$
L_{1} \otimes L_{2} \otimes L_{3}=\mathcal{O}_{\tilde{X}}
$$

Then we define $\tilde{V}$ as a sequence of extensions

$$
\begin{aligned}
& 0 \longrightarrow L_{1} \longrightarrow \tilde{W} \longrightarrow L_{2} \longrightarrow 0, \\
& 0 \longrightarrow \tilde{W} \longrightarrow \tilde{V} \longrightarrow L_{3} \longrightarrow 0 .
\end{aligned}
$$

Eq. (5.2) assures that the structure group of $\tilde{V}$ is $\mathrm{SU}(3)$ rather than $\Upsilon(3)$. The structure group of the rank 2 vector bundle $\tilde{W}$ is $\Upsilon(2)$. For $\tilde{V}$ to descend to the quotient manifold $X$, it has to be equivariant. To achieve that, it is sufficient to require that the line bundles $L_{1}, L_{2}, L_{3}$ are equivariant. A discussion of equivariant line bundles on the Schoen manifold can be found in [3]. The action of the discrete group in [3] was chosen to be different from ours in (3.5). However, the conclusions on equivariance are the same. Here we will simply state the conclusions, referring to [3] for additional details.

First, any equivariant line bundle $L$ on $\tilde{X}$ has to be constructed out of the invariant divisors in (3.16). That is, it has to be of the form

$$
L=\mathcal{O}_{\tilde{X}}\left(c_{1} \phi+c_{2} \tau_{1}+c_{3} \tau_{2}\right),
$$

where $c_{1}, c_{2}, c_{3}$ are integers. In addition, the sum $c_{2}+c_{3}$ has to be divisible by 3 . In our toy model, we will choose $L_{1}, L_{2}, L_{3}$ to be

$$
\begin{aligned}
& L_{1}=\mathcal{O}_{\tilde{X}}\left(-2 \phi+2 \tau_{1}+\tau_{2}\right), \\
& L_{2}=\mathcal{O}_{\tilde{X}}\left(\tau_{1}-\tau_{2}\right), \\
& L_{3}=\mathcal{O}_{\tilde{X}}\left(2 \phi-3 \tau_{1}\right) .
\end{aligned}
$$


Note that eq. (5.2) is satisfied. We will take the trivial choice of the equivariant structure; that is, we will assume that the moduli space of $V$ given by (5.1) consists of the component of the moduli space of $\tilde{V}$ which is invariant under $\mathbb{Z}_{3} \times \mathbb{Z}_{3}$.

For $V$ to have structure group $\mathrm{SU}(3)$ rather than its subgroup, we have to make sure that there exist non-trivial extensions in (5.3). The spaces of non-trivial extensions are given by

$$
H^{1}\left(\tilde{X}, L_{1} \otimes L_{2}^{*}\right) \quad \text { and } \quad H^{1}\left(\tilde{X}, \tilde{W} \otimes L_{3}^{*}\right)
$$

respectively. For simplicity, we will often denote them by $[\tilde{W}]$ and $\left[\tilde{V}_{\tilde{W}}\right]$. Note that $H^{1}\left(\tilde{X}, \tilde{W} \otimes L_{3}^{*}\right)$ is the space of extensions for a fixed extension $\tilde{W}$ in $[\tilde{W}]$. That is why we denote it by $\left[\tilde{V}_{\tilde{W}}\right]$. Each element in the extension class defines a vector bundle. However, it is important to take into account that different elements in the extension class can define isomorphic vector bundles. Let $\tilde{W}_{1}$ and $\tilde{W}_{2}$ be two vector bundles from the same extension class $[\tilde{W}]$. That is, they both satisfy

$$
\begin{aligned}
& 0 \longrightarrow L_{1} \longrightarrow \tilde{W}_{1} \longrightarrow L_{2} \longrightarrow 0 \\
& 0 \longrightarrow L_{1} \longrightarrow \tilde{W}_{2} \longrightarrow L_{2} \longrightarrow 0
\end{aligned}
$$

For any line bundle $L$ there is an isomorphism $L \rightarrow \lambda L$, where we multiply all elements of the fiber of $L$ by a non-zero complex number $\lambda$. Let us consider the following isomorphisms of $L_{1}$ and $L_{2}: L_{1} \rightarrow L_{1}, L_{2} \rightarrow \lambda L_{2}$. Then from the "five" lemma (see e.g. [34]), it follows that $W_{1}$ and $W_{2}$ are isomorphic. This means that elements in $H^{1}\left(\tilde{X}, L_{1} \otimes L_{2}^{*}\right)$ related by a multiplication by $\lambda \in \mathbb{C}^{*}$ correspond to the same vector bundle. Therefore, the moduli space $\mathcal{M}(\tilde{W})$ of vector bundles corresponding to the extension class $[\tilde{W}]$ is the projectivization of $H^{1}\left(\tilde{X}, L_{1} \otimes L_{2}^{*}\right)$ :

$$
\mathcal{M}(\tilde{W})=\mathbb{P} H^{1}\left(\tilde{X}, L_{1} \otimes L_{2}^{*}\right) .
$$

Note that

$$
\operatorname{dim} \mathcal{M}(\tilde{W})=h^{1}\left(\tilde{X}, L_{1} \otimes L_{2}^{*}\right)-1 .
$$

Similarly, the moduli space $\mathcal{M}\left(\tilde{V}_{\tilde{W}}\right)$ of vector bundles corresponding to the extension class $\left[\tilde{V}_{\tilde{W}}\right]$ is given by

$$
\mathcal{M}\left(\tilde{V}_{\tilde{W}}\right)=\mathbb{P} H^{1}\left(\tilde{X}, \tilde{W} \otimes L_{3}^{*}\right), \quad \operatorname{dim} \mathcal{M}\left(\tilde{V}_{\tilde{W}}\right)=h^{1}\left(\tilde{X}, \tilde{W} \otimes L_{3}^{*}\right)-1 .
$$

The full moduli space $\mathcal{M}(\tilde{V})$ of $\tilde{V}$ can be then understood as a fibration over $\mathcal{M}(\tilde{W})=$ $\mathbb{P} H^{1}\left(\tilde{X}, L_{1} \otimes L_{2}^{*}\right)$, where the fiber at a fixed extension $\tilde{W}$ is given by $\mathcal{M}\left(\tilde{V}_{\tilde{W}}\right)=\mathbb{P} H^{1}(\tilde{X}, \tilde{W} \otimes$ $\left.L_{3}^{*}\right)$. In appendix B, we compute the dimensions of the spaces in (5.6). We find

$$
h^{1}\left(\tilde{X}, L_{1} \otimes L_{2}^{*}\right)=18, \quad h^{1}\left(\tilde{X}, \tilde{W} \otimes L_{3}^{*}\right)=117 .
$$

This means that

$$
\operatorname{dim} \mathcal{M}(\tilde{W})=17, \quad \operatorname{dim} \mathcal{M}\left(\tilde{V}_{\tilde{W}}\right)=116
$$

and, hence,

$$
\operatorname{dim} \mathcal{M}(\tilde{V})=17+116=133 .
$$


Note that if we introduce coordinates in the vector space of extensions, it is straightforward to introduce coordinates on its projectivization; that is, we simply treat these coordinates as homogeneous ones.

Since the line bundles $L_{1}, L_{2}, L_{3}$ are equivariant, they descend to the quotient manifold $X$. To simplify notation, we will denote the corresponding line bundles on $X$ by the same letters $L_{1}, L_{2}, L_{3}$. Hence, the vector bundles $W$ and $V$ on $X$, obtained by modding out $\tilde{W}$ and $\tilde{V}$ by the action of $\mathbb{Z}_{3} \times \mathbb{Z}_{3}$, can be defined by the similar extension sequences on $X$

$$
\begin{aligned}
& 0 \longrightarrow L_{1} \longrightarrow W \longrightarrow L_{2} \longrightarrow 0, \\
& 0 \longrightarrow W \longrightarrow V \longrightarrow L_{3} \longrightarrow 0 .
\end{aligned}
$$

As we mentioned before, we will take the trivial choice of the equivariant structure. This means that the the extension classes $[W]$ and $\left[V_{W}\right]$ can be taken to be the invariant components of (5.6). That is,

$$
h^{1}\left(X, L_{1} \otimes L_{2}^{*}\right)=2, \quad h^{1}\left(X, W \otimes L_{3}^{*}\right)=13,
$$

where we have simply divided the dimensions in (5.11) by the order of $\mathbb{Z}_{3} \times \mathbb{Z}_{3}$. Then it follows that

$$
\operatorname{dim} \mathcal{M}(W)=1, \quad \operatorname{dim} \mathcal{M}\left(V_{W}\right)=12, \quad \operatorname{dim} \mathcal{M}(V)=1+12=13 .
$$

To show that $\tilde{V}$ and, hence, $V$ admits an Hermitian connection satisfying eq. (2.8), we need to prove that the extensions described above correspond to stable vector bundles. This is discussed in appendix $\mathrm{C}$.

\subsection{The ambient space description of $\tilde{V}$}

As was shown in the previous section, the line bundles $\mathcal{O}_{\tilde{X}}(\phi), \mathcal{O}_{\tilde{X}}\left(\tau_{1}\right), \mathcal{O}_{\tilde{X}}\left(\tau_{2}\right)$ can be obtained as restrictions of line bundles on the ambient space. Using (3.27), we find that $L_{1}, L_{2}, L_{3}$ are also restrictions of line bundles on $\mathcal{A}$. Let us define

$$
\mathcal{L}_{1}=\mathcal{O}_{\mathcal{A}}(-2,2,1), \quad \mathcal{L}_{2}=\mathcal{O}_{\mathcal{A}}(0,1,-1), \quad \mathcal{L}_{3}=\mathcal{O}_{\mathcal{A}}(2,-3,0) .
$$

Then $L_{1}=\left.\mathcal{L}_{1}\right|_{\tilde{X}}, L_{2}=\left.\mathcal{L}_{2}\right|_{\tilde{X}}, L_{3}=\left.\mathcal{L}_{3}\right|_{\tilde{X}}$. This implies that the extensions $\tilde{W}$ and $\tilde{V}$ are also restrictions of extensions on $\mathcal{A}$, which we denote by $\tilde{\mathcal{W}}$ and $\tilde{\mathcal{V}}$ respectively. They satisfy

$$
\begin{aligned}
& 0 \longrightarrow \mathcal{L}_{1} \longrightarrow \tilde{\mathcal{W}} \longrightarrow \mathcal{L}_{2} \longrightarrow 0, \\
& 0 \longrightarrow \tilde{\mathcal{W}} \longrightarrow \tilde{\mathcal{V}} \longrightarrow \mathcal{L}_{3} \longrightarrow 0
\end{aligned}
$$

Let us denote by $[\tilde{\mathcal{W}}]$ and $\left[\tilde{\mathcal{V}}_{\tilde{\mathcal{W}}}\right]$ the extension classes whose elements form the vector spaces

$$
H^{1}\left(\mathcal{A}, \mathcal{L}_{1} \otimes \mathcal{L}_{2}^{*}\right)=H^{1}\left(\mathcal{A}, \mathcal{O}_{\mathcal{A}}(-2,1,2)\right) \quad \text { and } \quad H^{1}\left(\mathcal{A}, \tilde{\mathcal{W}} \otimes \mathcal{L}_{3}^{*}\right)
$$

Similarly to our discussion in the previous subsection, we can introduce the moduli spaces of the corresponding vector bundles

$$
\begin{aligned}
\mathcal{M}(\tilde{\mathcal{W}}) & =\mathbb{P} H^{1}\left(\mathcal{A}, \mathcal{L}_{1} \otimes \mathcal{L}_{2}^{*}\right), & \operatorname{dim} \mathcal{M}(\tilde{\mathcal{W}}) & =h^{1}\left(\mathcal{A}, \mathcal{L}_{1} \otimes \mathcal{L}_{2}^{*}\right)-1, \\
\mathcal{M}\left(\tilde{\mathcal{V}}_{\tilde{\mathcal{W}}}\right) & =\mathbb{P} H^{1}\left(\mathcal{A}, \tilde{\mathcal{W}} \otimes \mathcal{L}_{3}^{*}\right), & \operatorname{dim} \mathcal{M}\left(\tilde{\mathcal{V}}_{\tilde{\mathcal{W}}}\right) & =h^{1}\left(\mathcal{A}, \tilde{\mathcal{W}} \otimes \mathcal{L}_{3}^{*}\right)-1
\end{aligned}
$$


Let us now study how the spaces (5.19), (5.20) are related to the similar spaces in (5.6), (5.8), (5.10). The dimension of the first cohomology group in (5.19) can be computed using the Kunneth formula and the Bott's formula

$$
h^{q}\left(\mathbb{P}^{n}, \mathcal{O}_{\mathbb{P}^{n}}(k)\right)= \begin{cases}\frac{(n+k) !}{n ! k !} & \text { for } q=0, n \geq 0, k \geq 0 \\ \frac{(-k-1) !}{n !(-k-n-1) !} & \text { for } q=n, n \geq 0, k \leq-(n+1) . \\ 0 & \text { otherwise }\end{cases}
$$

We then find that

$$
h^{1}\left(\mathcal{A}, \mathcal{L}_{1} \otimes \mathcal{L}_{2}^{*}\right)=18
$$

Comparing with (5.11), we observe that $h^{1}\left(\tilde{X}, L_{1} \otimes L_{2}^{*}\right)=h^{1}\left(\mathcal{A}, \mathcal{L}_{1} \otimes \mathcal{L}_{2}^{*}\right)=18$. This means that all extensions in $[\tilde{\mathcal{W}}]$ descend to non-trivial extensions on $\tilde{X}$, and that all extensions in $[\tilde{W}]$ are obtained as a restriction of extensions in $[\tilde{\mathcal{W}}]$. The dimension of the second cohomology group in (5.19) can be obtained by tensoring the first line in $(5.18)$ with $\mathcal{L}_{3}^{*}$ to get

$$
0 \longrightarrow \mathcal{O}_{\mathcal{A}}(-4,5,1) \longrightarrow \tilde{\mathcal{W}} \otimes \mathcal{L}_{3}^{*} \longrightarrow \mathcal{O}_{\mathcal{A}}(-2,4,-1) \longrightarrow 0
$$

Now the dimension of $H^{1}\left(\mathcal{A}, \tilde{\mathcal{W}} \otimes \mathcal{L}_{3}^{*}\right)$ can be computed from the cohomology long exact sequence corresponding to (5.23), again using the Kunneth and Bott's formulas. We find that

$$
h^{1}\left(\mathcal{A}, \tilde{\mathcal{W}} \otimes \mathcal{L}_{3}^{*}\right)=189 .
$$

Comparing this with eq. $(5.11)$, we see that $h^{1}\left(\mathcal{A}, \tilde{\mathcal{W}} \otimes \mathcal{L}_{3}^{*}\right)>h^{1}\left(\tilde{X}, \tilde{W} \otimes L_{3}^{*}\right)$. This means that $189-117=72$ non-trivial extensions of $\left[\tilde{\mathcal{V}}_{\tilde{\mathcal{W}}}\right]$ on $\mathcal{A}$ get restricted to zero on $\tilde{X}$.

Let us now describe the space of extensions on $\tilde{X}$ in terms of the cohomology groups on the ambient space. Cohomology groups on projective spaces can be written in terms of polynomials. Hence, this way we will obtain an explicit polynomial representation of the elements of $[\tilde{W}]$ and $\left[\tilde{V}_{\tilde{W}}\right]$. Furthermore, taking those polynomials which are invariant under $\mathbb{Z}_{3} \times \mathbb{Z}_{3}$ will give us an explicit polynomial parametrization of $[W]$ and $\left[V_{W}\right]$. Taking the projectivization of the corresponding vector spaces will give us a parametrization of the moduli spaces $\mathcal{M}(W)$ and $\mathcal{M}\left(V_{W}\right)$.

The relation between cohomology groups on $\mathcal{A}$ and on $\tilde{X} \subset \mathcal{A}$ can be obtained using the Koszul sequence - as we now explain. The Calabi-Yau threefold $\tilde{X}$ is defined as a submanifold in $\mathcal{A}$ using eqs. (3.3). Since $\tilde{X}$ is of co-dimension 2, its normal bundle is a rank 2 vector bundle. From eqs. (3.3) we find that it is a restriction of the following vector bundle on $\mathcal{A}$ :

$$
\mathcal{N}=\mathcal{N}_{1} \oplus \mathcal{N}_{2}, \quad \mathcal{N}_{1}=\mathcal{O}_{\mathcal{A}}(1,3,0), \quad \mathcal{N}_{2}=\mathcal{O}_{\mathcal{A}}(1,0,3)
$$

Let $\mathcal{L}$ be a vector bundle on $\mathcal{A}$ and $L=\left.\mathcal{L}\right|_{\tilde{X}}$. They are related to each other by the Koszul sequence

$$
0 \longrightarrow \wedge^{2} \mathcal{N}^{*} \otimes \mathcal{L} \stackrel{F^{\prime}}{\longrightarrow} \mathcal{N}^{*} \otimes \mathcal{L} \stackrel{F}{\longrightarrow} \mathcal{L} \stackrel{r}{\longrightarrow} L \longrightarrow 0 .
$$


The map $r$ is the restriction map, the map $F$ is multiplication (from the left) by the row vector $\left(F_{1}, F_{2}\right)$ of the defining polynomials in (3.3) and the map $F^{\prime}$ is determined by the composition rule $F \circ F^{\prime}=0$, which follows from the exactness of (5.26). This implies that $F^{\prime}$ is a column vector $\left(F_{2},-F_{1}\right)^{T}$. Note that the sequence (5.26) is not short and, hence, we cannot write the long exact cohomology sequence directly. However, one can split (5.26) into two short exact sequences by introducing an auxiliary sheaf $\mathcal{S}$ :

$$
\begin{aligned}
& 0 \longrightarrow \wedge^{2} \mathcal{N}^{*} \otimes \mathcal{L} \stackrel{F^{\prime}}{\longrightarrow} \mathcal{N}^{*} \otimes \mathcal{L} \stackrel{H_{1}}{\longrightarrow} \mathcal{S} \longrightarrow 0, \\
& 0 \longrightarrow \mathcal{S} \stackrel{H_{2}}{\longrightarrow} \mathcal{L} \stackrel{r}{\longrightarrow} L \longrightarrow 0
\end{aligned}
$$

where the maps $H_{1}, H_{2}$ satisfy $H_{2} \circ H_{1}=F$. Writing the long exact cohomology sequences for (5.27) allows us to compute the cohomology groups of $L$ in terms of the cohomology groups of $\mathcal{L}, \mathcal{N}^{*} \otimes \mathcal{L}$ and $\wedge^{2} \mathcal{N}^{*} \otimes \mathcal{L}$. These, in turn, can be calculated using the Kunneth and Bott formulas. We will not present the details of these laborious calculations and only give the results.

To compute the space of extensions $[\tilde{W}]$ we apply the Koszul sequence $(5.25)$ to $L=$ $L_{1} \otimes L_{2}^{*}$. Then we find that

$$
H^{1}\left(\tilde{X}, L_{1} \otimes L_{2}^{*}\right)=H^{1}\left(\mathcal{A}, \mathcal{L}_{1} \otimes \mathcal{L}_{2}^{*}\right)=H^{1}\left(\mathcal{A}, \mathcal{O}_{\mathcal{A}}(-2,1,2)\right)
$$

To compute the space of extensions $\left[\tilde{V}_{\tilde{W}}\right]$, we apply the Koszul sequence (5.26) to $L=$ $\tilde{W} \otimes L_{3}^{*}$. But first we have to find the cohomology groups of $\tilde{\mathcal{W}} \otimes \mathcal{L}_{3}^{*}, \tilde{\mathcal{W}} \otimes \mathcal{L}_{3}^{*} \otimes \mathcal{N}^{*}$ and $\tilde{\mathcal{W}} \otimes \mathcal{L}_{3}^{*} \otimes \wedge^{2} \mathcal{N}^{*}$ using (5.18). We obtain

$$
\begin{aligned}
h^{\bullet}\left(\mathcal{A}, \tilde{\mathcal{W}} \otimes \mathcal{L}_{3}^{*}\right) & =h^{\bullet}\left(\mathcal{A}, \mathcal{O}_{\mathcal{A}}(-4,5,1)\right)=(0,189,0,0,0,0), \\
h^{\bullet}\left(\mathcal{A}, \tilde{\mathcal{W}} \otimes \mathcal{N}^{*} \otimes \mathcal{L}_{3}^{*}\right) & =(0,72,0,36,0,0) \\
h^{1}\left(\mathcal{A}, \tilde{\mathcal{W}} \otimes \mathcal{N}^{*} \otimes \mathcal{L}_{3}^{*}\right) & =h^{1}\left(\mathcal{A}, \mathcal{O}_{\mathcal{A}}(-5,2,1)\right)=72 \\
h^{3}\left(\mathcal{A}, \tilde{\mathcal{W}} \otimes \mathcal{N}^{*} \otimes \mathcal{L}_{3}^{*}\right) & =h^{3}\left(\mathcal{A}, \mathcal{O}_{\mathcal{A}}(-3,4,-4)\right)=36 \\
h^{\bullet}\left(\mathcal{A}, \tilde{\mathcal{W}} \otimes \wedge^{2} \mathcal{N}^{*} \otimes \mathcal{L}_{3}^{*}\right) & =h^{\bullet}\left(\mathcal{A}, \mathcal{O}_{\mathcal{A}}(-4,1,-4)\right)=(0,0,0,27,0,0)
\end{aligned}
$$

Now, from the Koszul sequence (5.26) applied to $L=\tilde{W} \otimes L_{3}^{*}$ we find

$$
\begin{aligned}
& H^{1}\left(\tilde{X}, \tilde{W} \otimes L_{3}^{*}\right)=\frac{H^{1}\left(\mathcal{A}, \tilde{\mathcal{W}} \otimes \mathcal{L}_{3}^{*}\right)}{F_{1} \cdot H^{1}\left(\mathcal{A}, \tilde{\mathcal{W}} \otimes \mathcal{N}^{*} \otimes \mathcal{L}_{3}^{*}\right)}=\frac{H^{1}\left(\mathcal{A}, \mathcal{O}_{\mathcal{A}}(-4,5,1)\right)}{F_{1} \cdot H^{1}\left(\mathcal{A}, \mathcal{O}_{\mathcal{A}}(-5,2,1)\right)} \\
& h^{1}\left(\tilde{X}, \tilde{W} \otimes L_{3}^{*}\right)=189-72=117
\end{aligned}
$$

Here $F_{1}$ is the first defining polynomial in (3.3). It can be viewed as an element of $H^{0}\left(\mathcal{A}, \mathcal{O}_{\mathcal{A}}(1,3,0)\right)$. When we multiply $F_{1}$ by a differential in $H^{1}\left(\mathcal{A}, \mathcal{O}_{\mathcal{A}}(-5,2,1)\right)$, we naturally obtain a differential in $H^{1}\left(\mathcal{A}, \mathcal{O}_{\mathcal{A}}(-4,5,1)\right)$. Eq. (5.32) simply means that to find the extension class $\left[\tilde{V}_{\tilde{W}}\right]$ on $\tilde{X}$ we have to mod out by the image of the map $F_{1}$. The elements in the image of $F_{1}$, that is, the denominator in (5.32), are precisely the extensions on the ambient space which do not correspond to extensions on $\tilde{X}$. From eq. (5.32) we see that they become zero when we restrict to $\tilde{X}$, since $F_{1}$ vanishes on $\tilde{X}$. 
Note that the right hand side of eq. (5.32) does not depend on the choice of an element $\tilde{W}$ in $[\tilde{W}]$ or in $\mathcal{M}(\tilde{W})$. This means that the moduli space $\mathcal{M}(\tilde{V})$ is the trivial fibration

$$
\mathcal{M}(\tilde{V})=\mathcal{M}(\tilde{W}) \times \mathcal{M}\left(\tilde{V}_{\tilde{W}}\right)
$$

where the first factor is the projectivization of the vector space in (5.28) and the second factor is the projectivization of the vector space in (5.32).

\subsection{Parametrization of the moduli space}

The aim of this subsection is to derive a parameterization of the moduli spaces $\mathcal{M}\left(\tilde{V}_{\tilde{W}}\right)$ and $\mathcal{M}\left(V_{W}\right)$. Parametrization of the moduli spaces of extensions $\mathcal{M}(\tilde{W})$ and $\mathcal{M}(W)$ can be derived in a similar way, but is not required in this paper.

First, we consider the numerator in (5.32). According to the Kunneth and Bott formulas,

$$
H^{1}\left(\mathcal{A}, \mathcal{O}_{\mathcal{A}}(-4,5,1)\right)=H^{1}\left(\mathbb{P}^{1}, \mathcal{O}_{\mathbb{P}^{1}}(-4)\right) \otimes H^{0}\left(\mathbb{P}^{2} \times \mathbb{P}^{2}, \mathcal{O}_{\mathbb{P}^{2} \times \mathbb{P}^{2}}(5,1)\right)
$$

Let us consider the vector space

$$
H^{1}\left(\mathbb{P}^{1}, \mathcal{O}_{\mathbb{P}^{1}}(-3)\right) \simeq H^{0}\left(\mathbb{P}^{1}, \mathcal{O}_{\mathbb{P}^{1}}(1)\right)^{*}
$$

where we have used Serre duality. This vector space is 2-dimensional and we denote its basis as $\left\{r_{0}, r_{1}\right\}$. This basis is chosen to be dual to the basis $\left\{t_{0}, t_{1}\right\}$ of homogeneous degree 1 polynomials on $\mathbb{P}^{1}$. The vector space of interest,

$$
H^{1}\left(\mathbb{P}^{1}, \mathcal{O}_{\mathbb{P}^{1}}(-4)\right) \simeq H^{0}\left(\mathbb{P}^{1}, \mathcal{O}_{\mathbb{P}^{1}}(2)\right)^{*}
$$

is 3 -dimensional with a natural basis $\left\{r_{0}^{2}, r_{0} r_{1}, r_{1}^{2}\right\}$ dual to the basis $\left\{t_{0}^{2}, t_{0} t_{1}, t_{1}^{2}\right\}$ of degree 2 polynomials on $\mathbb{P}^{1}$. It follows that an arbitrary element $v \in H^{1}\left(\mathcal{A}, \mathcal{O}_{\mathcal{A}}(-4,5,1)\right)$ can be written as

$$
v=r_{0}^{2} f_{1}(\mathbf{x}, \mathbf{y})+r_{0} r_{1} f_{2}(\mathbf{x}, \mathbf{y})+r_{1}^{2} f_{3}(\mathbf{x}, \mathbf{y})
$$

where $f_{1}, f_{2}, f_{3}$ are homogeneous polynomials on $\mathbb{P}^{2} \times \mathbb{P}^{2}$ of degree $(5,1)$. Here, to simplify our notation, we let $\mathbf{x}$ denote the coordinates on the first $\mathbb{P}^{2}, \mathbf{x} \equiv\left[x_{0}: x_{1}: x_{2}\right]$ and, similarly, y denotes the coordinates on the second $\mathbb{P}^{2}, \mathbf{y} \equiv\left[y_{0}: y_{1}: y_{2}\right]$. The coefficients in the polynomials $f_{1}, f_{2}, f_{3}$ can be viewed as coordinates on $H^{1}\left(\mathcal{A}, \mathcal{O}_{\mathcal{A}}(-4,5,1)\right)$. As we computed in (5.29), there are 189 such coefficients.

Since eventually we are interested in the moduli space of the vector bundle $V$ on $X$, we restrict $H^{1}\left(\mathcal{A}, \mathcal{O}_{\mathcal{A}}(-4,5,1)\right)$ to its subspace consisting of elements $v_{i n v}$ which are invariant under $\mathbb{Z}_{3} \times \mathbb{Z}_{3}$. Since the discrete group does not act on $\mathbb{P}^{1}$ (see eq. (3.5)), the elements $r_{0}$ and $r_{1}$ are automatically invariant. Hence, $v_{i n v}$ is of the form (5.37) where the polynomials $f_{1}, f_{2}, f_{3}$ are restricted to be the $\mathbb{Z}_{3} \times \mathbb{Z}_{3}$ invariant polynomials of degree $(5,1)$. Let us 
introduce a basis for these invariant polynomials:

$$
\begin{aligned}
& E_{1}=x_{0}^{5} y_{0}+x_{1}^{5} y_{1}+x_{2}^{5} y_{2}, \\
& E_{2}=x_{0}^{2} x_{1}^{3} y_{0}+x_{1}^{2} x_{2}^{3} y_{1}+x_{2}^{2} x_{0}^{3} y_{2}, \\
& E_{3}=x_{0}^{2} x_{2}^{3} y_{0}+x_{1}^{2} x_{0}^{3} y_{1}+x_{2}^{2} x_{1}^{3} y_{2}, \\
& E_{4}=x_{0}^{2} x_{1} x_{2} y_{0}+x_{1}^{2} x_{2} x_{0} y_{1}+x_{2}^{2} x_{0} x_{1} y_{2}, \\
& E_{5}=x_{1}^{4} x_{2} y_{0}+x_{2}^{4} x_{0} y_{1}+x_{0}^{4} x_{1} y_{2}, \\
& E_{6}=x_{0}^{5} y_{0}+x_{1}^{5} y_{1}+x_{2}^{5} y_{2}, \\
& E_{7}=x_{1} x_{2}^{4} y_{0}+x_{2} x_{0}^{4} y_{1}+x_{0} x_{1}^{4} y_{2} .
\end{aligned}
$$

The invariant polynomials $f_{1}, f_{2}, f_{3}$ are then given by

$$
f_{1}=\sum_{\alpha=1}^{7} a_{\alpha} E_{\alpha}, \quad f_{2}=\sum_{\alpha=1}^{7} b_{\alpha} E_{\alpha}, \quad f_{3}=\sum_{\alpha=1}^{7} c_{\alpha} E_{\alpha},
$$

where $\left(a_{\alpha}, b_{\alpha}, c_{\alpha}\right)$ are coordinates on the 21(=189/9)-dimensional invariant subspace of $H^{1}\left(\mathcal{A}, \mathcal{O}_{\mathcal{A}}(-4,5,1)\right)$. However, to obtain the invariant part of the extension class $\left[\tilde{V}_{\tilde{W}}\right]$ we have to mod out by those elements which can be obtained by multiplying the defining polynomial $F_{1}$ by elements of $H^{1}\left(\mathcal{A}, \mathcal{O}_{\mathcal{A}}(-5,2,1)\right)$. We discuss this in detail in appendix D. Here we simply state the result. Dividing by the the denominator in (5.32) is equivalent to imposing the following constraints on the coordinates $\left(a_{\alpha}, b_{\alpha}, c_{\alpha}\right)$ :

$$
\begin{aligned}
a_{1}+a_{2}+a_{3} & =0, & a_{4}+a_{5}+a_{6} & =0, \\
a_{4}+b_{1}+b_{2}+b_{3} & =0, & a_{7}+b_{4}+b_{5}+b_{6} & =0, \\
b_{4}+c_{1}+c_{2}+c_{3} & =0, & b_{7}+c_{4}+c_{5}+c_{6} & =0, \\
c_{4} & =0, & c_{7} & =0 .
\end{aligned}
$$

We can choose

$$
a_{1}, a_{2}, a_{5}, b_{1}, b_{2}, b_{3}, b_{5}, b_{6}, c_{1}, c_{2}, c_{3}, c_{5}, c_{6}
$$

as independent parameters, with the others being determined using eqs. (5.40). The parameters in (5.41) can be viewed as the 13 coordinates on the invariant subspace of the vector space of extensions. Note that this is consistent with (5.16). According to our previous discussion, the moduli space $\mathcal{M}\left(V_{W}\right)$ is obtained by projectivization of this vector space. This simply means that we should view the coordinates $\left(a_{\alpha}, b_{\alpha}, c_{\alpha}\right)$ as homogeneous ones.

To conclude this section, let us summarize the structure of the moduli space of $V$. It is given by the trivial fibration

$$
\mathcal{M}(V)=\mathcal{M}(W) \times \mathcal{M}\left(V_{W}\right)
$$

where

$$
\mathcal{M}(W)=\mathbb{P}^{1}, \quad \mathcal{M}\left(V_{W}\right)=\mathbb{P}^{12} .
$$

In total, we have 13 moduli of $V$. The parametrization of the second factor, $\mathcal{M}\left(V_{W}\right)$, is explicitly given by 21 homogenous coordinates $\left(a_{1}, \ldots, a_{7}, b_{1}, \ldots, b_{7}, c_{1}, \ldots, c_{7}\right)$ subject to 
8 linear constraints (5.40). We can choose 13 independent variables, as in eq. (5.41), and view them as 13 homogeneous coordinates on $\mathcal{M}\left(V_{W}\right)$. By similar methods, we can obtain a parametrization of $\mathcal{M}(W)$ - but we do not need it in this paper.

\section{The superpotential on $X$}

In this section, we will compute the leading non-perturbative superpotential, that is, $\sim$ $p=e^{i T^{1}}$ (see eq. (3.30)), in a heterotic string vacuum specified by $(X, V)$. To simplify our analysis, we will perform the calculations for fixed complex structure. Then $\left(\operatorname{det} \bar{\partial}_{\mathcal{O}_{C_{i}}}(-1)\right)$ in (2.22) become numerical constants which will not play any role and will be ignored. Our aim in this section will be to compute the Pfaffians. First, we will calculate them on the covering space $\tilde{X}$ and then on the quotient space $X$. Since we would like to compare these two calculations, in the theory on $\tilde{X}$ we will restrict ourselves to the invariant component of the moduli space which will descend to $X$. The method of computing the Pfaffians will be similar to the one introduced in [21,22]. Since we do not know either the metric or the connection, we will rely on an algebraic approach whose essence is to understand under which conditions each Pfaffian vanishes. The conditions will be derived as a homogeneous polynomial equation on the moduli space. Since the Pfaffian is a section of a line bundle on the moduli space and the moduli space is a projective space, this polynomial will be the Pfaffian up to a numerical coefficient which cannot be determined by our algebraic method.

Let us now review the general condition for the vanishing of a Pfaffian on a holomorphic, isolated, genus 0 curve $C[15,21,22]$. The Pfaffian vanishes if and only if the operator $\bar{\partial}_{V_{C}(-1)}$ has a zero mode. The zero modes of $\bar{\partial}$ are elements of the Dolbeault cohomology group. In the present case, the cohomology group of interest is $H^{0}\left(C,\left.V\right|_{C} \otimes \mathcal{O}_{C}(-1)\right)$. Hence, the Pfaffian vanishes if and only if $h^{0}\left(C,\left.V\right|_{C} \otimes \mathcal{O}_{C}(-1)\right) \neq 0$. Since $h^{0}\left(C,\left.V\right|_{C} \otimes \mathcal{O}_{C}(-1)\right)$ is not a topological invariant, it depends on where we are in the moduli space of $V$. For generic values of the moduli, $h^{0}\left(C,\left.V\right|_{C} \otimes \mathcal{O}_{C}(-1)\right)$ will be zero and $\bar{\partial}_{V_{C}(-1)}$ will not have zero modes. However, at a specific co-dimension 1 subspace of the moduli space $h^{0}\left(C,\left.V\right|_{C} \otimes \mathcal{O}_{C}(-1)\right)$ will jump - thus producing a zero mode. The Pfaffian of $\bar{\partial}_{V_{C}(-1)}$ will be determined by the equation defining this co-dimension 1 subspace.

\subsection{Calculation of the Pfaffians}

As was discussed in section 3.4, on $\tilde{X}$ there are 81 isolated curves of interest. These curves split into 9 orbits under the action of $\mathbb{Z}_{3} \times \mathbb{Z}_{3}$ with 9 curves in each orbit. If we restrict ourselves to the invariant part of the moduli space, all curves in the same orbit will give an identical contribution. Hence, in this case we need to compute the Pfaffians of the Dirac operator on any 9 curves which do not lie in the orbits of each other. An example of such curves was given in eqs. (3.41), (3.42), (3.10). Let us recall that these curves lie in different homology classes and, hence, have different areas measured by the Kahler form on $\tilde{X}$. However, they have the same area when measured using the invariant part of the Kahler form $\left.\omega_{\mathcal{A}}\right|_{\tilde{X}}$. Therefore, the images of these curves in $X$ have the same area with respect to the Kahler form $\omega_{X}$. 
Let us now study under which conditions $h^{0}\left(C,\left.\tilde{V}\right|_{C} \otimes \mathcal{O}_{C}(-1)\right) \neq 0$ for the curves $C$ of the type $\mathbb{P}^{1} \times \mathbf{x} \times \mathbf{y} \subset \tilde{X} \subset \mathbb{P}^{1} \times \mathbb{P}^{2} \times \mathbb{P}^{2}$. We denote $\mathcal{B}=\mathbb{P}^{2} \times \mathbb{P}^{2}$ and define $p_{\mathcal{B}}$ to be the projection $p_{\mathcal{B}}: \mathcal{A} \rightarrow \mathcal{B}$ with fibers being $\mathbb{P}^{1}$. Now consider a particular extension element $\tilde{\mathcal{W}}$ on $\mathcal{A}$,

$$
0 \longrightarrow \mathcal{L}_{1} \longrightarrow \tilde{\mathcal{W}} \longrightarrow \mathcal{L}_{2} \longrightarrow 0,
$$

and tensor this sequence with $\mathcal{O}_{\mathcal{A}}(-1,0,0)$ to obtain

$$
0 \longrightarrow \mathcal{O}_{\mathcal{A}}(-3,2,1) \longrightarrow \tilde{\mathcal{W}}(-1,0,0) \longrightarrow \mathcal{O}_{\mathcal{A}}(-1,1,1) \longrightarrow 0
$$

where we have used eqs. (5.17) and defined $\tilde{\mathcal{W}}(-1,0,0)=\tilde{\mathcal{W}} \otimes \mathcal{O}_{\mathcal{A}}(-1,0,0)$. Now take the direct image of this sequence with the projection $p_{\mathcal{B}}$. This leads to the exact sequence

$$
\begin{aligned}
0 \longrightarrow p_{\mathcal{B} *} \mathcal{O}_{\mathcal{A}}(-3,2,1) & \longrightarrow p_{\mathcal{B} *} \tilde{\mathcal{W}}(-1,0,0) \longrightarrow p_{\mathcal{B} *} \mathcal{O}_{\mathcal{A}}(-1,1,1) \longrightarrow \\
R^{1} p_{\mathcal{B}_{*}} \mathcal{O}_{\mathcal{A}}(-3,2,1) & \longrightarrow R^{1} p_{\mathcal{B} *} \tilde{\mathcal{W}}(-1,0,0) \longrightarrow R^{1} p_{\mathcal{B}_{*}} \mathcal{O}_{\mathcal{A}}(-1,1,1) \longrightarrow 0
\end{aligned}
$$

At each point on $\mathcal{B}$ and for any line bundle $\mathcal{L}, p_{\mathcal{B} *} \mathcal{L}$ is generated by the cohomology group of the fiber at this point; that is by $H^{0}\left(\mathbb{P}^{1},\left.\mathcal{L}\right|_{\mathbb{P}^{1}}\right)$. Similarly, $R^{1} p_{\mathcal{B} *} \mathcal{L}$ is generated by $H^{1}\left(\mathbb{P}^{1},\left.\mathcal{L}\right|_{\mathbb{P}^{1}}\right)$. Clearly, for $\mathcal{L}$ of the form $\mathcal{L}=\mathcal{O}_{\mathcal{A}}\left(m_{1}, m_{2}, m_{3}\right)$, we have $\left.\mathcal{L}\right|_{\mathbb{P}^{1}}=\mathcal{O}_{\mathbb{P}^{1}}\left(m_{1}\right)$. Then, using the Bott's formula, we compute that

$$
\begin{aligned}
p_{\mathcal{B} *} \mathcal{O}_{\mathcal{A}}(-3,2,1) & =0, \quad p_{\mathcal{B} *} \mathcal{O}_{\mathcal{A}}(-1,1,1)=0, \quad R^{1} p_{\mathcal{B} *} \mathcal{O}_{\mathcal{A}}(-1,1,1)=0, \\
R^{1} p_{\mathcal{B} *} \mathcal{O}_{\mathcal{A}}(-3,2,1) & =H^{1}\left(\mathbb{P}^{1}, \mathcal{O}_{\mathbb{P}^{1}}(-3)\right) \otimes \mathcal{O}_{\mathcal{B}}(2,1)
\end{aligned}
$$

Therefore, from eq. (6.3) we obtain

$$
\begin{aligned}
p_{\mathcal{B} *} \tilde{\mathcal{W}}(-1,0,0) & =0 \\
R^{1} p_{\mathcal{B} *} \tilde{\mathcal{W}}(-1,0,0) & =R^{1} p_{\mathcal{B} *} \mathcal{O}_{\mathcal{A}}(-3,2,1)=H^{1}\left(\mathbb{P}^{1}, \mathcal{O}_{\mathbb{P}^{1}}(-3)\right) \otimes \mathcal{O}_{\mathcal{B}}(2,1) .
\end{aligned}
$$

Note that the right hand side in eqs. (6.5) is independent of the choice of the extension representative $\tilde{\mathcal{W}}$.

As the next step, we consider the sequence defining $\tilde{\mathcal{V}}$ tensored with $\mathcal{O}_{\mathcal{A}}(-1,0,0)$. Using eqs. (5.17), (5.18) we obtain

$$
0 \longrightarrow \tilde{\mathcal{W}}(-1,0,0) \longrightarrow \tilde{\mathcal{V}}(-1,0,0) \longrightarrow \mathcal{O}_{\mathcal{A}}(1,-3,0) \longrightarrow 0,
$$

where $\tilde{\mathcal{V}}(-1,0,0)=\tilde{\mathcal{V}} \otimes \mathcal{O}_{\mathcal{A}}(-1,0,0)$. Taking the direct image of this sequence with the projection $p_{\mathcal{B}}$ and using (6.5) we obtain

$$
\begin{aligned}
0 \longrightarrow \quad p_{\mathcal{B} *} \tilde{\mathcal{V}}(-1,0,0) & \longrightarrow \\
H^{0}\left(\mathbb{P}^{1}, \mathcal{O}_{\mathbb{P}^{1}}(1)\right) \otimes \mathcal{O}_{\mathcal{B}}(-3,0) & \stackrel{\delta(\tilde{\mathcal{V}})}{\longrightarrow} H^{1}\left(\mathbb{P}^{1}, \mathcal{O}_{\mathbb{P}^{1}}(-3)\right) \otimes \mathcal{O}_{\mathcal{B}}(2,1) \longrightarrow \\
R^{1} p_{\mathcal{B} *} \tilde{\mathcal{V}}(-1,0,0) & \longrightarrow 0 .
\end{aligned}
$$

Here $\delta(\tilde{\mathcal{V}})$ is a map depending on the moduli of $\tilde{\mathcal{V}}$. Note that the vector spaces $H^{0}\left(\mathbb{P}^{1}, \mathcal{O}_{\mathbb{P}^{1}}(1)\right)$ and $H^{1}\left(\mathbb{P}^{1}, \mathcal{O}_{\mathbb{P}^{1}}(-3)\right)$ are 2-dimensional, which implies that $\delta(\tilde{\mathcal{V}})$ can be represented by a $2 \times 2$ matrix. 
Let us now consider the sequence (6.7) at any point $\mathbf{x} \times \mathbf{y} \subset \mathcal{B}$ which corresponds to a curve $C=\mathbb{P}^{1} \times \mathbf{x} \times \mathbf{y} \subset \mathcal{A}$. This curve is also a curve in $\tilde{X}$ if the point $\mathbf{x} \times \mathbf{y}$ satisfies equations (3.38). At any point $\mathbf{x} \times \mathbf{y} \in \mathcal{B}, p_{\mathcal{B} *} \tilde{\mathcal{V}}(-1,0,0)$ is generated by the cohomology group $H^{0}\left(C,\left.\tilde{\mathcal{V}}\right|_{C} \otimes \mathcal{O}_{C}(-1)\right)$. If $\mathbf{x} \times \mathbf{y}$ is chosen to satisfy eq. (3.38), then we also have $H^{0}\left(C,\left.\tilde{\mathcal{V}}\right|_{C} \otimes \mathcal{O}_{C}(-1)\right)=H^{0}\left(C,\left.\tilde{V}\right|_{C} \otimes \mathcal{O}_{C}(-1)\right)$ - which is precisely the space of zero modes of the Dirac operator. This space is non-empty if and only if the map $\left.\delta(\tilde{\mathcal{V}})\right|_{\mathbf{x} \times \mathbf{y}}$ has a non-trivial kernel. Let us define $\delta(\tilde{V})=\left.\delta(\tilde{\mathcal{V}})\right|_{\tilde{X}}$. Note that if $\mathbf{x} \times \mathbf{y}$ corresponds to a curve in $\tilde{X}$, then we have $\left.\delta(\tilde{V})\right|_{\mathbf{x} \times \mathbf{y}}=\left.\delta(\tilde{\mathcal{V}})\right|_{\mathbf{x} \times \mathbf{y}}$. If we represent $\left.\delta(\tilde{V})\right|_{\mathbf{x} \times \mathbf{y}}$ by a $2 \times 2$ matrix, it then follows that

$$
\operatorname{Pfaff}_{\tilde{X}}\left(\bar{\partial}_{V_{C_{i}}(-1)}\right)=0 \quad \text { if and only if } \quad \operatorname{det}\left[\left.\delta(\tilde{V})\right|_{\mathbf{x} \times \mathbf{y}}\right]=0 .
$$

Let us now construct the matrix $\delta(\tilde{V})$ which provides a map $\delta(\tilde{V}): \mathcal{H}_{1} \rightarrow \mathcal{H}_{2}$, where we denoted

$$
\begin{aligned}
& \mathcal{H}_{1}=H^{0}\left(\mathbb{P}^{1}, \mathcal{O}_{\mathbb{P}^{1}}(1)\right) \otimes \mathcal{O}_{\mathcal{B}}(-3,0), \\
& \mathcal{H}_{2}=H^{1}\left(\mathbb{P}^{1}, \mathcal{O}_{\mathbb{P}^{1}}(-3)\right) \otimes \mathcal{O}_{\mathcal{B}}(2,1) .
\end{aligned}
$$

Recall from eq. (5.32) that modulo the denominator - which will be taken into account later - the space of extensions is given by the elements

$$
v \in H^{1}\left(\mathcal{A}, \mathcal{O}_{\mathcal{A}}(-4,5,1)\right)=H^{0}\left(\mathbb{P}^{1}, \mathcal{O}_{\mathbb{P}^{1}}(-4)\right) \otimes H^{1}\left(\mathcal{B}, \mathcal{O}_{\mathcal{B}}(5,1)\right),
$$

where $v$ can explicitly be written as (see (5.37))

$$
v=r_{0}^{2} f_{1}(\mathbf{x}, \mathbf{y})+r_{0} r_{1} f_{2}(\mathbf{x}, \mathbf{y})+r_{1}^{2} f_{3}(\mathbf{x}, \mathbf{y})
$$

Comparing eq. (6.9) and eq. (6.10), we conclude that $\delta(\tilde{V})$ is given by multiplication by $v$. As was discussed around eq. (5.35), we can introduce the basis $\left\{t_{0}, t_{1}\right\}$ for $H^{0}\left(\mathbb{P}^{1}, \mathcal{O}_{\mathbb{P}^{1}}(1)\right)$ and the dual basis $\left\{r_{0}, r_{1}\right\}$ for $H^{1}\left(\mathbb{P}^{1}, \mathcal{O}_{\mathbb{P}^{1}}(-3)\right)$. To construct the matrix $\delta(\tilde{V})$, we simply multiply $v$ by the basis elements $\left\{t_{0}, t_{1}\right\}$ to get

$$
v\left(t_{0}\right)=r_{0} f_{1}+r_{1} f_{2}, \quad v\left(t_{1}\right)=r_{0} f_{2}+r_{1} f_{3}
$$

and present the answer in the matrix form

$$
\delta(\tilde{V})\left(\begin{array}{c}
r_{0} \\
r_{1}
\end{array}\right) .
$$

This gives

$$
\delta(\tilde{V})=\left(\begin{array}{ll}
f_{1} & f_{2} \\
f_{2} & f_{3}
\end{array}\right), \quad \operatorname{det}[\delta(\tilde{V})]=f_{1} f_{3}-f_{2}^{2} .
$$

If $C$ is a curve corresponding to a specific point $\mathbf{x} \times \mathbf{y} \in \mathbb{P}^{2} \times \mathbb{P}^{2}$ then we get

$$
\operatorname{det}\left[\left.\delta(\tilde{V})\right|_{\mathbf{x} \times \mathbf{y}}\right]=\left(f_{1} f_{3}-f_{2}^{2}\right)(\mathbf{x}, \mathbf{y}) .
$$


After we evaluate $f_{1} f_{3}-f_{2}^{2}$ at points of $\mathbb{P}^{2} \times \mathbb{P}^{2}$, the right hand side of (6.15) becomes a degree 2 homogeneous polynomial of the parameters of $f_{1}, f_{2}, f_{3}$. For the purposes of our paper, we can restrict $f_{1}, f_{2}, f_{3}$ to be the invariant polynomials under $\mathbb{Z}_{3} \times \mathbb{Z}_{3}$. They are explicitly given by eqs. (5.38), (5.39). Furthermore, we recall that to describe the extensions of $\tilde{V}$ rather than those of $\tilde{\mathcal{V}}$, we have to impose the relations (5.40). We also recall that to describe the moduli space of $\tilde{\mathcal{V}}$, we projectivize the corresponding space of extensions. This simply means that we view the parameters $\left(a_{\alpha}, b_{\alpha}, c_{\alpha}\right)$ of the polynomials $f_{1}, f_{2}, f_{3}$ as homogeneous coordinates. Since the moduli space is a projective space and $\left(f_{1} f_{3}-f_{2}^{2}\right)(\mathbf{x}, \mathbf{y})$ is a homogeneous polynomial of degree 2 , we conclude that (6.15) is a section of a line bundle of degree 2 on the moduli space. Finally, we notice that eq. (6.15) depends only on the coordinates of $\mathcal{M}\left(\tilde{V}_{\tilde{W}}\right)$. The coordinates of $\mathcal{M}(\tilde{W})$ drop out from our calculations.

From eq. (6.8) and the fact the Pfaffian is a section of a line bundle on the moduli space, we conclude that

$$
\operatorname{Pfaff}_{\tilde{X}}\left(\bar{\partial}_{V_{C}(-1)}\right) \sim\left(f_{1} f_{3}-f_{2}^{2}\right)(\mathbf{x}, \mathbf{y})=\sum_{\alpha, \beta=1}^{7}\left(a_{\alpha} c_{\beta}-b_{\alpha} b_{\beta}\right) E_{\alpha} E_{\beta}(\mathbf{x}, \mathbf{y})
$$

up to a numerical coefficient - which we are not able to compute by our method. Let us now apply this result to the curves (3.41), (3.43). Denote

$$
\mathcal{R}_{\tilde{X}, i}=\left(f_{1} f_{3}-f_{2}^{2}\right)\left(s_{i}\right), \quad i=1, \ldots, 9 .
$$

Substituting the points (3.41), (3.43) into (6.17), we obtain the following expressions for $\mathcal{R}_{i}\left(\zeta=e^{2 \pi i / 3}\right)$ :

$$
\begin{aligned}
& \mathcal{R}_{\tilde{X}, 1}=-\left(2 b_{1}-b_{2}-b_{3}\right)^{2}+\left(2 a_{1}-a_{2}-a_{3}\right)\left(2 c_{1}-c_{2}-c_{3}\right), \\
& \mathcal{R}_{\tilde{X}, 2}=-\left(b_{2}+b_{3} \zeta^{2}+b_{1} \zeta\right)^{2}+\left(a_{2}+a_{3} \zeta^{2}+a_{1} \zeta\right)\left(c_{2}+c_{3} \zeta^{2}+c_{1} \zeta\right), \\
& \mathcal{R}_{\tilde{X}, 3}=-\left(b_{2}+b_{3} \zeta+b_{1} \zeta^{2}\right)^{2}+\left(a_{2}+a_{3} \zeta+a_{1} \zeta^{2}\right)\left(c_{2}+c_{3} \zeta^{2}+c_{1} \zeta\right), \\
& \mathcal{R}_{\tilde{X}, 4}=-\left(-b_{1}+b_{3}+b_{5}-b_{6}\right)^{2}+\left(-a_{1}+a_{3}+a_{5}-a_{6}\right)\left(-c_{1}+c_{3}+c_{5}-c_{6}\right), \\
& \mathcal{R}_{\tilde{X}, 5}=-\left(-b_{1}+b_{2}-b_{5}+b_{6}\right)^{2}+\left(-a_{1}+a_{2}-a_{5}+a_{6}\right)\left(-c_{1}+c_{2}-c_{5}+c_{6}\right), \\
& \mathcal{R}_{\tilde{X}, 6}=-\left(-b_{1}+b_{3}+\left(b_{5}-b_{6}\right) \zeta^{2}\right)^{2}+\left(-a_{1}+a_{3}+\left(a_{5}-a_{6}\right) \zeta^{2}\right)\left(-c_{1}+c_{3}+\left(c_{5}-c_{6}\right) \zeta^{2}\right), \\
& \mathcal{R}_{\tilde{X}, 7}=-\left(-b_{1}+b_{2}-\left(b_{5}-b_{6}\right) \zeta^{2}\right)^{2}+\left(-a_{1}+a_{2}-\left(a_{5}-a_{6}\right) \zeta^{2}\right)\left(-c_{1}+c_{2}-\left(c_{5}-c_{6}\right) \zeta^{2}\right), \\
& \mathcal{R}_{\tilde{X}, 8}=-\left(-b_{1}+b_{2}-\left(b_{5}-b_{6}\right) \zeta\right)^{2}+\left(-a_{1}+a_{2}-\left(a_{5}-a_{6}\right) \zeta\right)\left(-c_{1}+c_{2}-\left(c_{5}-c_{6}\right) \zeta\right), \\
& \mathcal{R}_{\tilde{X}, 9}=-\left(-b_{1}+b_{3}+\left(b_{5}-b_{6}\right) \zeta\right)^{2}+\left(-a_{1}+a_{3}+\left(a_{5}-a_{6}\right) \zeta\right)\left(-c_{1}+c_{3}+\left(c_{5}-c_{6}\right) \zeta\right) .
\end{aligned}
$$

The parameters $\left(a_{\alpha}, b_{\alpha}, c_{\alpha}\right)$ satisfy the relations (5.40), but substituting them into (6.18) does not lead to a simplification. Note that none of the polynomials $\mathcal{R}_{\tilde{X}, i}$ depends on the parameters $a_{4}, a_{7}, b_{4}, b_{7}, c_{4}, c_{7}$. The reason is because the corresponding polynomials $E_{4}$ and $E_{7}$ in (5.38) vanish on any curve satisfying eqs. (3.38).

Let us now introduce the proportionality coefficient into (6.16). For each of our 9 curves, we denote it by $A_{\tilde{X}, i}$ where $i=1, \ldots, 9$. That is, we have

$$
\operatorname{Pfaff}_{\tilde{X}}\left(\bar{\partial}_{\tilde{V}_{C_{i}}(-1)}\right)=A_{\tilde{X}, i} \mathcal{R}_{\tilde{X}, i} .
$$


Note that every $A_{\tilde{X}, i}$ is non-zero because the Pfaffians vanish only along the zero locus of the polynomials $\mathcal{R}_{\tilde{X}, i}$ and do not vanish identically. We are not able to compute the coefficients $A_{\tilde{X}, i}$ by our algebraic method. However, it is possible to constrain them using the Beasley-Witten residue theorem, which we will now discuss.

\subsection{The residue theorem on $\tilde{X}$}

Our theory on $\tilde{X}$ formally satisfies the assumptions of Beasley and Witten in [23], which we reviewed in subsection 2.2. Indeed, the Calabi-Yau threefold $\tilde{X}$ is, by construction, a projective complete intersection manifold and the vector bundle $\tilde{V}$ is the restriction of a vector bundle $\tilde{\mathcal{V}}$. Nevertheless, as we discussed in subsection 2.3 , the residue theorem of Beasley-Witten is not directly applicable here since $h^{1,1}(\tilde{X})>h^{1,1}(\mathcal{A})$. However, indirectly we can still apply it. If we measure the area of curves in $\tilde{X}$ using the $(1,1)$ form $\left.\omega_{\mathcal{A}}\right|_{\tilde{X}}$ then, according to the residue theorem, the sum of the Pfaffians of all curves with the same area has to vanish. The 81 curves found in subsection 3.4 have the same area with respect to $\left.\omega_{\mathcal{A}}\right|_{\tilde{X}}$ and, hence, we can apply the residue theorem to them. Since we are restricting ourselves to the invariant part of the moduli space, all curves in the same $\mathbb{Z}_{3} \times \mathbb{Z}_{3}$ orbit will have an identical Pfaffian. This means that it is enough to sum the Pfaffians of the curves which do not lie in the orbit of each other. These Pfaffians are given in eqs. (6.18) and (6.19). Hence, the residue theorem implies that

$$
\sum_{i=1}^{9} A_{\tilde{X}, i} \mathcal{R}_{\tilde{X}, i}=0 .
$$

Let us stress again that eq. (6.20) does not imply that the superpotentail in the heterotic theory on $\tilde{X}$ vanishes because in (6.20) we are summing the Pfaffians of curves lying in different homology classes and having different area with respect to the proper Kahler form $\omega_{\tilde{X}}$. Hence, in the superpotential these Pfaffians will be weighted with different exponential prefactors and cannot cancel each other. Eq. (6.20) constrains the coefficients $A_{\tilde{X}, i}$. It is possible to satisfy eq. (6.20) if and only if the polynomials $\mathcal{R}_{\tilde{X}, i}$ in (6.18) are linearly dependent - which is a non-trivial consistency check on our calculations. It is possible to check (using e.g. Mathematica) that these polynomials are indeed linearly dependent and it possible to adjust the parameters $A_{\tilde{X}, i}$ so that the sum in (6.20) vanishes. More precisely, requiring that the sum in (6.20) vanish puts the following constraints on $A_{\tilde{X}, i}$ :

$$
\begin{aligned}
& A_{\tilde{X}, 1}=-A_{\tilde{X}, 4}-A_{\tilde{X}, 5}, \\
& A_{\tilde{X}, 2}=e^{i \pi / 3} A_{\tilde{X}, 4}-A_{\tilde{X}, 7}, \\
& A_{\tilde{X}, 3}=-e^{i \pi / 3} A_{\tilde{X}, 5}+e^{-i \pi / 3}\left(A_{\tilde{X}, 4}-A_{\tilde{X}, 7}\right), \\
& A_{\tilde{X}, 6}=A_{\tilde{X}, 4}+A_{\tilde{X}, 5}-A_{\tilde{X}, 7}, \\
& A_{\tilde{X}, 8}=e^{i \pi / 3} A_{\tilde{X}, 5}-e^{2 i \pi / 3} A_{\tilde{X}, 7}, \\
& A_{\tilde{X}, 9}=A_{\tilde{X}, 4}+e^{-i \pi / 3}\left(A_{\tilde{X}, 5}-A_{\tilde{X}, 7}\right) .
\end{aligned}
$$

These results will play a role in constructing the superpotential on the quotient Calabi-Yau manifold $X$. 


\subsection{The explicit formula for the superpotential on $X$}

Since the curves $C_{i}$ corresponding to solutions (3.41), (3.43) lie in different $\mathbb{Z}_{3} \times \mathbb{Z}_{3}$ orbits, they descend to 9 different curves on $X$. To simplify our notation, we will still denote these curves by $C_{i}$. The non-perturbative superpotential for these curves is then given by

$$
W_{X}([C])=e^{i T^{1}} \sum_{i=1}^{9} \operatorname{Pfaff}_{X}\left(\bar{\partial}_{V_{C_{i}}(-1)}\right) \chi_{i} .
$$

As was discussed in section 3 , these 9 curves lie in the same homology class in $H_{2}(X, \mathbb{R})$ but in 9 different homology classes in $H_{2}(X, \mathbb{Z})$. This means that the contribution for each curve will pick up a distinct $\mathbb{Z}_{3} \times \mathbb{Z}_{3}$ character $\chi_{i}$. As we discussed in subsection 2.4 , as long as the curves in the same homology class in $H_{2}(X, \mathbb{Z})$ receive the same character and curves in different homology classes receive different characters, the distribution of the characters among the curves is arbitrary and depends on the choice of the connected component of the moduli space of the $B$-field.

To compute $W_{X}([C])$ in $(6.22)$, we notice that since the Pfaffians in the previous subsection were computed for the invariant part of the moduli space. It follows that

$$
\operatorname{Pfaff}_{X}\left(\bar{\partial}_{V_{C_{i}}(-1)}\right)=\operatorname{Pfaff}_{\tilde{X}}\left(\bar{\partial}_{\tilde{V}_{C_{i}}(-1)}\right)
$$

where the right hand side is given by eqs. (6.19), (6.18), (6.21). In particular, it follows that

$$
\operatorname{Pfaff}_{X}\left(\bar{\partial}_{V_{C_{i}}(-1)}\right)=A_{X, i} \mathcal{R}_{X, i}, \quad \mathcal{R}_{X, i}=\mathcal{R}_{\tilde{X}, i}, \quad A_{X, i}=A_{\tilde{X}, i} .
$$

The reason is that, by construction, the gauge connection on $V$ is the equivariant connection on $\tilde{V}$. For the trivial choice of the equivariant structure, it is just the connection on $\tilde{V}$ restricted to the invariant part of the moduli space. Since in the previous subsection we restricted our calculations to the invariant part of the moduli space, the Dirac operators on both sides in (6.23) depend on the same connection and, hence, their Pfaffians are equal. Thus, the superpotential in (6.22) becomes

$$
W_{X}([C])=e^{i T^{1}} \sum_{i=1}^{9} \chi_{i} A_{X, i} \mathcal{R}_{X, i},
$$

where $\mathcal{R}_{X, i}$ are also given by (6.18) and $A_{X, i}$ also satisfy the constraints (6.21). Now, the key observation is that, since the linear combination $\sum_{i=1}^{9} A_{X, i} \mathcal{R}_{X, i}=0$ due to the residue theorem, the linear combination in (6.25) $\sum_{i=1}^{9} \chi_{i} A_{X, i} \mathcal{R}_{X, i}$ is non-zero because it is twisted by the characters $\chi_{i}$, most of which are not unity.

As an example let us give some assignment of the characters to the curves. Note that though 9 different characters $\chi_{i}$ label 9 inequivalent representations of $\mathbb{Z}_{3} \times \mathbb{Z}_{3}$, they can take only 3 values in $\mathbb{C}^{*}$ given by $1, e^{2 \pi i / 3}, e^{4 \pi i / 3}$. Assigning, for example,

$$
\chi_{1}=\chi_{2}=\chi_{3}=1 \quad \chi_{4}=\chi_{5}=\chi_{6}=e^{2 \pi i / 3}, \quad \chi_{7}=\chi_{8}=\chi_{9}=e^{4 \pi i / 3}
$$

we obtain

$$
W_{X}([C])=e^{i T^{1}}\left(\sum_{i=1}^{3} A_{X, i} \mathcal{R}_{X, i}+e^{2 \pi i / 3} \sum_{i=4}^{6} A_{X, i} \mathcal{R}_{X, i}+e^{4 \pi i / 3} \sum_{i=7}^{9} \mathcal{R}_{X, i}\right) .
$$


It is straightforward to check using eqs. (6.18) and (6.21) that the linear combination (6.27) does not vanish. From eqs. (5.40), (5.41), (6.18) we see that $W_{X}([C])$ depends on 12 out of the 13 moduli of $\mathcal{M}(V)$. The remaining modulus parametrizing $\mathcal{M}(W)$ does not show up in the superpotential (6.27). Our expression for $W_{X}([C])$ depends on 9 numerical coefficients $A_{X, i}$ which we cannot fully compute by our algebraic method. However, due to relations (6.21), only 3 of them are really unknown.

Thus, we have explicitly demonstrated that in our model on $X$ a non-vanishing, nonperturbative superpotential can be generated in the low-energy field theory.

\section{Conclusion and future directions}

In this paper, we presented examples of heterotic string compactifications with nonvanishing non-perturbative superpotentials. In our examples, the superpotential does not vanish on both the simply connected covering space and the non-simply connected manifold obtained as a quotient by the action of the discrete isometry group. In both cases, the reason for the non-vanishing of the superpotential can be attributed to the existence of holomorphic, isolated, genus 0 curves which are unique in their integer homology classes.

It would be interesting to generalize the ideas developed in this paper for realistic heterotic models and to compute non-perturbative superpotentials in a heterotic MSSM. The heterotic Standard Model constructed in [1-4] used a different Schoen manifold with a different action of $\mathbb{Z}_{3} \times \mathbb{Z}_{3}$. Hence, it would be interesting to see if one can build a heterotic MSSM on the Schoen manifold used in this paper. Then one can extend the results of this paper to compute the non-perturbative superpotential in an MSSM, rather than in a toy model. The result is expected to be non-zero, as in our present examples.

Another possible direction is to apply our methods to realistic heterotic models obtained using the monad construction [5-9, 33]. The crucial difference is that such models are built on projective Calabi-Yau manifolds satisfying $h^{1,1}(\tilde{X})=h^{1,1}(\mathcal{A})$. Then, according to the Beasley-Witten residue theorem, the non-perturbative superpotential vanishes on the covering manifold $\tilde{X}$. However, on the quotient manifold $X$ it might be nonzero because the second homology group of $X$ is expected to contain discrete torsion. It would be interesting to see if one indeed can generate a non-perturbative superpotential in such models.

\section{Acknowledgments}

The authors are very grateful to Tony Pantev for valuable discussions. The authors would also like to thank Ling Lin for helpful conversations. The work of E. I. Buchbinder was supported by the ARC Future Fellowship FT120100466 and in part by the ARC Discovery project DP140103925. B. A. Ovrut is supported in part by the DOE under contract No. DE-SC0007901. E.I.B. would like to thank the physics department at the University of Pennsylvania where some of this work was done for warm hospitality. 


\section{A The normal bundle to the curves in $\tilde{X}$}

Here we will compute the normal bundle to the curves in subsection 3.4. Specifically, we present our calculations for the curve specified by $s_{1}$ in eq. (3.41). The other curves can be treated similarly and give the same result.

The curve $s_{1}$ is of the form

$$
C_{1}=\left[t_{0}: t_{1}\right] \times s_{1}=\left[t_{0}, t_{1}\right] \times[1:-1: 0] \times[1,-1,0] .
$$

Let us first consider the short exact sequence relating the tangent bundle $T \tilde{X}$ and the normal bundle $N \tilde{X}$ of $\tilde{X}$; that is

$$
\left.0 \longrightarrow T \tilde{X} \stackrel{h_{2}}{\longrightarrow} T \mathcal{A}\right|_{\tilde{X}} \stackrel{h_{1}}{\longrightarrow} N \tilde{X} \longrightarrow 0,
$$

where $T \mathcal{A}$ is the tangent bundle of the ambient space given by

$$
T \mathcal{A}=\mathcal{O}_{\mathbb{P}^{1}}(2) \oplus T \mathbb{P}^{2} \oplus T \mathbb{P}^{2}
$$

and we have used the fact that the tangent bundle of $\mathbb{P}^{1}$ is $\mathcal{O}_{\mathbb{P}^{1}}(2)$. Using eqs. (3.3), the normal bundle $N \tilde{X}$ is

$$
N \tilde{X}=\left.\left.\mathcal{O}_{\mathcal{A}}(1,3,0)\right|_{\tilde{X}} \oplus \mathcal{O}_{\mathcal{A}}(1,0,3)\right|_{\tilde{X}} .
$$

We now want to restrict the sequence (A.2) to the curve $C_{1}$. For the curve of the form (A.1), we obtain

$$
\left.T \mathcal{A}\right|_{C_{1}}=\mathcal{O}_{C_{1}}(2) \oplus \mathcal{O}_{C_{1}}^{4},\left.\quad N \tilde{X}\right|_{C_{1}}=\mathcal{O}_{C_{1}}(1) \oplus \mathcal{O}_{C_{1}}(1) .
$$

The sequence (A.2) then becomes

$$
\left.0 \longrightarrow T \tilde{X}\right|_{C_{1}} \stackrel{\left.h_{2}\right|_{C_{1}}}{\longrightarrow} \mathcal{O}_{C_{1}}(2) \oplus \mathcal{O}_{C_{1}}^{4} \stackrel{\left.h_{1}\right|_{C_{1}}}{\longrightarrow} \mathcal{O}_{C_{1}}(1) \oplus \mathcal{O}_{C_{1}}(1) \longrightarrow 0 .
$$

Let us now analyze the maps $h_{1}$ and $h_{2}$. The map $h_{1}$ is defined as a map from tangent directions $\partial$ along $\mathcal{A}$ to the column vector $\left(\partial F_{1}, \partial F_{2}\right)^{T}$. Since $T \mathcal{A}$ is of rank 5 and $N \tilde{X}$ is of rank $2, h_{1}$ is a $2 \times 5$ matrix. Evaluating the derivatives of $F_{1}$ and $F_{2}$ and restricting the results to the curve (A.1) gives

$$
\left.h_{1}\right|_{C_{1}}=\left(\begin{array}{ccccc}
0 & 3 t_{0} & t_{1} & 0 & 0 \\
0 & 0 & 0 & 3\left(\lambda_{1} t_{0}+t_{1}\right) & \lambda_{2} t_{0}+\lambda_{3} t_{1}
\end{array}\right) .
$$

Since the sequence (A.6) is exact, it follows that $h_{1}$ and $h_{2}$ satisfy the composition rule $h_{1} \circ h_{2}=0$. This determines $\left.h_{2}\right|_{C_{1}}$ to be

$$
\left.h_{2}\right|_{C_{1}}=\left(\begin{array}{ccc}
1 & 0 & 0 \\
0 & t_{1} & 0 \\
0 & -3 t_{0} & 0 \\
0 & 0 & \lambda_{2} t_{0}+\lambda_{3} t_{1} \\
0 & 0 & -3\left(\lambda_{1} t_{0}+t_{1}\right)
\end{array}\right)
$$

up to an arbitrary holomorphic section $h_{0}$ on $C_{1}$ which is a homogeneous polynomials of degree $k \geq 0$ in $\left[t_{0}: t_{1}\right]$. Since any vector bundle on $C_{1} \simeq \mathbb{P}^{1}$ is a sum of line bundles, 
$\left.T \tilde{X}\right|_{C_{1}}$ must be of the form $\mathcal{O}_{C_{1}}\left(m_{1}\right) \oplus \mathcal{O}_{C_{1}}\left(m_{2}\right) \oplus \mathcal{O}_{C_{1}}\left(m_{3}\right)$ where, from (A.6), it follows that $m_{1}+m_{2}+m_{3}=0$. Examining the sequence (A.6), it is easy to see that these conditions have only one consistent possibility, namely $m_{1}=2, m_{2}=m_{3}=-1$. This means that

$$
\left.T \tilde{X}\right|_{C_{1}}=\mathcal{O}_{C_{1}}(2) \oplus \mathcal{O}_{C_{1}}(-1) \oplus \mathcal{O}_{C_{1}}(-1) .
$$

Finally, let us consider the short exact sequence relating the tangent bundle $T C_{1}$ and the normal bundle $N C_{1}$ of $C_{1}$ given by

$$
\left.0 \longrightarrow T C_{1} \longrightarrow T \tilde{X}\right|_{C_{1}} \longrightarrow N C_{1} \longrightarrow 0 \text {. }
$$

Using eq. (A.9), we obtain

$$
0 \longrightarrow \mathcal{O}_{C_{1}}(2) \longrightarrow \mathcal{O}_{C_{1}}(2) \oplus \mathcal{O}_{C_{1}}(-1) \oplus \mathcal{O}_{C_{1}}(-1) \longrightarrow N C_{1} \longrightarrow 0
$$

This implies that the only possible form for $N C_{1}$ is

$$
N C_{1}=\mathcal{O}_{C_{1}}(-1) \oplus \mathcal{O}_{C_{1}}(-1)
$$

\section{B Extension of $\tilde{W}$ and $\tilde{V}$}

In this appendix, we calculate the number of extensions of $\tilde{W}$ and $\tilde{V}$ and prove eq. (5.11). Our calculations will be similar to the ones performed in [3], where additional details can be found.

\section{B.1 Extensions of $\tilde{W}$}

The extensions of $\tilde{W}$ are given by the dimension of the cohomology group

$$
H^{1}\left(\tilde{X}, L_{1} \otimes L_{2}^{*}\right)=H^{1}\left(\tilde{X}, \mathcal{O}_{\tilde{X}}\left(-2 \phi+\tau_{1}+2 \tau_{2}\right)\right)
$$

Let us consider the direct image $\pi_{1 *} L_{1} \otimes L_{2}^{*}$ under the projection $\pi_{1}$ in the diagram (3.10). Using the definitions of $\phi, \tau_{1}, \tau_{2}$ in (3.16), we can give $L_{1} \otimes L_{2}^{*}$ in the form

$$
L_{1} \otimes L_{2}^{*}=\pi_{1}^{*} \mathcal{O}_{B_{1}}(t-2 f) \otimes \pi_{2}^{*} \mathcal{O}_{B_{2}}(2 t) .
$$

From the diagram (3.10), it follows that the projections satisfy

$$
\pi_{1 *} \pi_{2}^{*}=\beta_{1}^{*} \beta_{2 *}
$$

Then we obtain

$$
\pi_{1 *} L_{1} \otimes L_{2}^{*}=\mathcal{O}_{B_{1}}(t-2 f) \otimes \beta_{1}^{*} \beta_{2 *} \mathcal{O}_{B_{2}}(2 t)=\beta_{1}^{*} \mathcal{O}_{\mathbb{P}^{1}}(-2) \otimes \mathcal{O}_{B_{1}}(t) \otimes \beta_{1}^{*} \beta_{2 *} \mathcal{O}_{B_{2}}(2 t)
$$

Computing $R^{1} \pi_{1 *} L_{1} \otimes L_{2}^{*}$, we find that

$$
R^{1} \pi_{1 *} L_{1} \otimes L_{2}^{*}=0
$$


To show this, note that at each point $p$ on $B_{1}, R^{1} \pi_{1 *} L_{1} \otimes L_{2}^{*}$ is generated by the first cohomology group

$$
H^{1}\left(F_{p},\left.\mathcal{O}_{\tilde{X}}\left(-2 \phi+\tau_{1}+2 \tau_{2}\right)\right|_{F_{p}}\right)
$$

on the elliptic fiber $F_{p}$ of the projection $\pi_{1}$ at $p$. Using eqs. (3.15), (3.16) we find that the line bundle $\left.\mathcal{O}_{\tilde{X}}\left(-2 \phi+\tau_{1}+2 \tau_{2}\right)\right|_{F_{p}}$ has degree 3 and by the Kodaira vanishing theorem (see e.g [44]) the cohomology group in (B.6) vanishes. This proves (B.5). As the next step we similarly project (B.4) to the base of $B_{1}$. We obtain

$$
\begin{aligned}
\beta_{1 *} \pi_{1 *} L_{1} \otimes L_{2}^{*} & =\mathcal{O}_{\mathbb{P}^{1}}(-2) \otimes \beta_{1 *} \mathcal{O}_{B_{1}}(t) \otimes \beta_{2 *} \mathcal{O}_{B_{2}}(2 t), \\
R^{1} \beta_{1 *} \pi_{1 *} L_{1} \otimes L_{2}^{*} & =0
\end{aligned}
$$

Using the identities [3]

$$
\beta_{k *} \mathcal{O}_{B_{k}}(t)=\mathcal{O}_{\mathbb{P}}^{\oplus 3}, \quad \beta_{k *} \mathcal{O}_{B_{k}}(2 t)=\mathcal{O}_{\mathbb{P}^{1}}^{\oplus 6}, \quad k=1,2
$$

we find that

$$
\beta_{1 *} \pi_{1 *} L_{1} \otimes L_{2}^{*}=\mathcal{O}_{\mathbb{P}^{1}}(-2)^{\oplus 18} .
$$

Since the higher direct images in eqs. (B.5), (B.8) vanish from a Leray spectral sequence, it follows that

$$
h^{1}\left(\tilde{X}, L_{1} \otimes L_{2}^{*}\right)=h^{1}\left(\mathbb{P}^{1}, \beta_{1 *} \pi_{1 *} L_{1} \otimes L_{2}^{*}\right)=h^{1}\left(\mathbb{P}^{1}, \mathcal{O}_{\mathbb{P}^{1}}(-2)^{\oplus 18}\right)=18 .
$$

\section{B.2 Extensions of $\tilde{V}$}

The number of extensions of $\tilde{V}$ (for a fixed extension $\tilde{W}$ in $[\tilde{W}]$ ) is given by $H^{1}\left(\tilde{X}, \tilde{W} \otimes L_{3}^{*}\right)$. To compute this cohomology group, we consider the short exact sequence

$$
0 \longrightarrow L_{1} \otimes L_{3}^{*} \longrightarrow \tilde{W} \otimes L_{3}^{*} \longrightarrow L_{2} \otimes L_{3}^{*} \longrightarrow 0,
$$

where

$$
L_{1} \otimes L_{3}^{*}=\mathcal{O}_{\tilde{X}}\left(-4 \phi+5 \tau_{1}+\tau_{2}\right), \quad L_{2} \otimes L_{3}^{*}=\mathcal{O}_{\tilde{X}}\left(-2 \phi+4 \tau_{1}-\tau_{2}\right) .
$$

The sequence (B.12) implies the following long exact sequence of cohomology groups

$$
\begin{aligned}
0 \longrightarrow & H^{0}\left(\tilde{X}, L_{1} \otimes L_{3}^{*}\right) \longrightarrow H^{0}\left(\tilde{X}, \tilde{W} \otimes L_{3}^{*}\right) \longrightarrow H^{0}\left(\tilde{X}, L_{2} \otimes L_{3}^{*}\right) \longrightarrow \\
& H^{1}\left(\tilde{X}, L_{1} \otimes L_{3}^{*}\right) \longrightarrow H^{1}\left(\tilde{X}, \tilde{W} \otimes L_{3}^{*}\right) \longrightarrow H^{1}\left(\tilde{X}, L_{2} \otimes L_{3}^{*}\right) \longrightarrow \\
& H^{2}\left(\tilde{X}, L_{1} \otimes L_{3}^{*}\right) \longrightarrow \ldots
\end{aligned}
$$

The cohomology of $L_{1} \otimes L_{3}^{*}$ and $L_{2} \otimes L_{3}^{*}$ can be computed using direct images, just as in the previous subsection. Using the identities [3]

$$
\begin{aligned}
\beta_{k *} \mathcal{O}_{B_{k}}(4 t) & =\mathcal{O}_{\mathbb{P}^{1}}^{\oplus 9} \oplus \mathcal{O}_{\mathbb{P}^{1}}(1)^{\oplus 3}, \\
\beta_{k *} \mathcal{O}_{B_{k}}(5 t) & =\mathcal{O}_{\mathbb{P}^{1}}^{\oplus 9} \oplus \mathcal{O}_{\mathbb{P}^{1}}(1)^{\oplus 6}, \\
R^{1} \beta_{k *} \mathcal{O}_{B_{k}}(-t) & =\mathcal{O}_{\mathbb{P}^{1}}(-1)^{\oplus 3}
\end{aligned}
$$


and following the same steps as in the previous subsection, we obtain

$$
\begin{aligned}
H^{0}\left(\tilde{X}, L_{1} \otimes L_{3}^{*}\right) & =H^{2}\left(\tilde{X}, L_{1} \otimes L_{3}^{*}\right)=0, \\
H^{1}\left(\tilde{X}, L_{1} \otimes L_{3}^{*}\right) & =H^{1}\left(\mathbb{P}^{1}, \beta_{1 *} \pi_{1 *} L_{1} \otimes L_{3}^{*}\right)=H^{1}\left(\mathbb{P}^{1}, \mathcal{O}_{\mathbb{P}^{1}}(-4)^{\oplus 27} \oplus \mathcal{O}_{\mathbb{P}^{1}}(-3)^{\oplus 18}\right), \\
h^{1}\left(\tilde{X}, L_{1} \otimes L_{3}^{*}\right) & =117, \\
H^{0}\left(\tilde{X}, L_{2} \otimes L_{3}^{*}\right) & =H^{1}\left(\tilde{X}, L_{2} \otimes L_{3}^{*}\right)=H^{2}\left(\tilde{X}, L_{2} \otimes L_{3}^{*}\right)=0 .
\end{aligned}
$$

Then from (B.14) we see that

$$
H^{1}\left(\tilde{X}, \tilde{W} \otimes L_{3}^{*}\right)=H^{1}\left(\tilde{X}, L_{1} \otimes L_{3}^{*}\right), \quad h^{1}\left(\tilde{X}, \tilde{W} \otimes L_{3}^{*}\right)=117 .
$$

\section{Stability of $\tilde{W}$ and $\tilde{V}$}

Since we are only considering a toy model, we will not give a comprehensive proof that $\tilde{W}$ and $\tilde{V}$ are stable. Instead, we examine the most important necessary condition for this to be the case.

Let us recall that a vector bundle $\tilde{V}$ on $\tilde{X}$ is called stable if for any subsheaf $S$ of lower rank we have

$$
\mu(S)<\mu(\tilde{V}) .
$$

Here, the slope $\mu(S)$ is defined by

$$
\mu(S)=\frac{1}{\operatorname{rk}(S)} \int_{\tilde{X}} c_{1}(S) \wedge \omega_{\tilde{X}} \wedge \omega_{\tilde{X}}
$$

where $\omega_{\tilde{X}}$ is the Kahler form on $\tilde{X}$.

From eqs. (5.3), we observe that the line bundle $L_{1}$ injects into $\tilde{W}$ and $\tilde{W}$ injects into $\tilde{V}$. We now discuss whether $L_{1}$ and $\tilde{W}$ destabilize $\tilde{W}$ and $\tilde{V}$ respectively. Using the definition of $\tilde{W}$ in (5.3), we see that $\tilde{W}$ has rank 2 and its first Chern class is given by $c_{1}\left(L_{1}\right)+c_{1}\left(L_{2}\right)$. Then the condition $\mu\left(L_{1}\right)<\mu(\tilde{W})$ can be stated as

$$
\int_{\tilde{X}}\left(c_{1}\left(L_{1}\right)-c_{1}\left(L_{2}\right)\right) \wedge \omega_{\tilde{X}} \wedge \omega_{\tilde{X}}<0 \quad \Leftrightarrow \quad \mu\left(L_{1} \otimes L_{2}^{*}\right)<0 .
$$

Since $L_{1}$ and $L_{2}$ are equivariant and constructed out of the invariant classes, we can replace $\omega_{\tilde{X}}$ in (C.3) with its invariant part $\omega_{X}$ in (3.18). Using the expression for the invariant part of the Kahler form in (3.18), we can rewrite (C.3) in the form

$$
\int_{\tilde{X}}\left(-2 \omega_{\phi}+\omega_{\tau_{1}}+2 \omega_{\tau_{2}}\right) \wedge\left(t^{1} \omega_{\phi}+t^{2} \omega_{\tau_{1}}+t^{3} \omega_{\tau_{2}}\right)^{2}<0 .
$$

Using the triple intersection numbers (3.17), we then obtain the following inequality for the Kahler parameters:

$$
\left(t^{3}\right)^{2}+4\left(t^{1}\right)^{2}+6 t^{1} t^{3}+24 t^{1} t^{2}-6 t^{2} t^{3}<0 .
$$


Let us now study the condition that $\mu(\tilde{W})<\mu(\tilde{V})$. Note that $c_{1}(\tilde{W})=c_{1}\left(L_{1}\right)+c_{1}\left(L_{2}\right)$ and, since $L_{1} \otimes L_{2} \otimes L_{3}$ is trivial, it follows that $c_{1}(\tilde{W})=c_{1}\left(L_{3}^{*}\right)$. Also note that since $c_{1}(\tilde{V})=0$, it follows that $\mu(\tilde{V})=0$. Then the condition $\mu(\tilde{W})<\mu(\tilde{V})$ can be stated as

$$
\mu\left(L_{3}^{*}\right)<0 \quad \Leftrightarrow \quad \int_{\tilde{X}}\left(-2 \omega_{\phi}+3 \omega_{\tau_{1}}\right) \wedge\left(t^{1} \omega_{\phi}+t^{2} \omega_{\tau_{1}}+t^{3} \omega_{\tau_{2}}\right)^{2}<0 .
$$

Using the triple intersection numbers (3.17), we then obtain the inequality

$$
\left(t^{3}\right)^{2}+6 t^{1} t^{3}-4 t^{2} t^{3}<0 .
$$

The bundles $\tilde{W}$ and $\tilde{V}$ are not destabilized if there exists a region in the Kahler moduli space where both inequalities (C.5) and (C.7) are simultaneously satisfied. It is easy to see that it is indeed the case. For example, if we take $t^{2} \approx t^{3}$ and $t^{1} \ll t^{2}, t^{3}$ both inequalities are satisfied.

\section{Parameterization of the moduli space of $V$}

Let us recall from section 5 that the invariant extensions in $[\tilde{V}]$, as well as the space of extensions $[V]$, are described by the invariant subspace of the quotient

$$
\frac{H^{1}\left(\mathcal{A}, \mathcal{O}_{\mathcal{A}}(-4,5,1)\right)}{F_{1} \cdot H^{1}\left(\mathcal{A}, \mathcal{O}_{\mathcal{A}}(-5,2,1)\right)}
$$

The elements of the numerator were parameterized as

$$
v_{i n v}=r_{0}^{2} f_{1}(\mathbf{x}, \mathbf{y})+r_{0} r_{1} f_{2}(\mathbf{x}, \mathbf{y})+r_{1}^{2} f_{3}(\mathbf{x}, \mathbf{y})
$$

where $f_{1}, f_{2}, f_{3}$ are invariant polynomials of degree $(5,1)$ on $\mathbb{P}^{2} \times \mathbb{P}^{2}$ and $\left\{r_{0}, r_{1}\right\}$ is a basis in the vector space $H^{1}\left(\mathbb{P}^{1}, \mathcal{O}_{\mathbb{P}^{1}}(-3)\right)$ dual to the basis $\left\{t_{0}, t_{1}\right\}$ in $H^{0}\left(\mathbb{P}^{1}, \mathcal{O}_{\mathbb{P}^{1}}(1)\right)$. The polynomials $f_{1}, f_{2}, f_{3}$ can be expanded in the basis (5.38)

$$
f_{1}=\sum_{\alpha=1}^{7} a_{\alpha} E_{\alpha}, \quad f_{2}=\sum_{\alpha=1}^{7} b_{\alpha} E_{\alpha}, \quad f_{3}=\sum_{\alpha=1}^{7} c_{\alpha} E_{\alpha} .
$$

The aim of this appendix is to describe the process of factoring out $F_{1} \cdot H^{1}\left(\mathcal{A}, \mathcal{O}_{\mathcal{A}}(-5,2,1)\right)$. This will give a parameterization of the invariant part of the moduli space of $\tilde{V}$ and of the moduli space of $V$.

Consider an element $u$ in $H^{1}\left(\mathcal{A}, \mathcal{O}_{\mathcal{A}}(-5,2,1)\right)$. Let us write it in the form similar to (D.2). Using the Kunneth and Bott formulas, we can express $H^{1}\left(\mathcal{A}, \mathcal{O}_{\mathcal{A}}(-5,2,1)\right)$ as

$$
H^{1}\left(\mathcal{A}, \mathcal{O}_{\mathcal{A}}(-5,2,1)\right)=H^{1}\left(\mathbb{P}^{1}, \mathcal{O}_{\mathbb{P}^{1}}(-5)\right) \otimes H^{0}\left(\mathbb{P}^{2} \times \mathbb{P}^{2}, \mathcal{O}_{\mathbb{P}^{2} \times \mathbb{P}^{2}}(2,1)\right) .
$$

In the first factor

$$
H^{1}\left(\mathbb{P}^{1}, \mathcal{O}_{\mathbb{P}^{1}}(-5)\right) \simeq H^{0}\left(\mathbb{P}^{1}, \mathcal{O}_{\mathbb{P}^{1}}(3)\right)^{*}
$$

we can introduce a natural basis $\left\{r_{0}^{3}, r_{0}^{2} r_{1}, r_{0} r_{1}^{2}, r_{1}^{3}\right\}$ dual to the basis $\left\{t_{0}^{3}, t_{0}^{2} t_{1}, t_{0} t_{1}^{2}, t_{1}^{3}\right\}$ of homogeneous polynomials of degree 3 in $H^{0}\left(\mathbb{P}^{1}, \mathcal{O}_{\mathbb{P}^{1}}(3)\right)$. Then $u$ can be written as

$$
u=r_{0}^{3} g_{1}(\mathbf{x}, \mathbf{y})+r_{0}^{2} r_{1} g_{2}(\mathbf{x}, \mathbf{y})+r_{0} r_{1}^{2} g_{3}(\mathbf{x}, \mathbf{y})+r_{1}^{3} g_{4}(\mathbf{x}, \mathbf{y}),
$$


where $g_{1}, g_{2}, g_{3}, g_{4}$ are homogeneous polynomials of degree $(2,1)$ on $\mathbb{P}^{2} \times \mathbb{P}^{2}$. To restrict to invariant elements $u_{i n v}$, we take $g_{1}, g_{2}, g_{3}, g_{4}$ to be invariant polynomials. The basis of invariant polynomials of degree $(2,1)$ can be chosen to be

$$
\begin{aligned}
& e_{1}=x_{0}^{2} y_{0}+x_{1}^{2} y_{1}+x_{2}^{2} y_{2}, \\
& e_{2}=x_{1} x_{2} y_{0}+x_{2} x_{0} y_{1}+x_{0} x_{1} y_{2} .
\end{aligned}
$$

Now let us consider the map $F_{1}$. Using eq. (3.3), we can write it in the form

$$
F_{1}=t_{0} \sigma_{0}(\mathbf{x})+t_{1} \sigma_{1}(\mathbf{x}) \quad \text { where } \quad \sigma_{0}(\mathbf{x})=x_{0} x_{1} x_{2}, \sigma_{1}(\mathbf{x})=x_{0}^{3}+x_{1}^{3}+x_{2}^{3} .
$$

Let us multiply $u_{\text {inv }}$ by $F_{1}$, using the fact that the bases $\left\{r_{0}, r_{1}\right\}$ and $\left\{t_{0}, t_{1}\right\}$ are dual to each other. We obtain

$$
F_{1} u_{i n v}=r_{0}^{2}\left(\sigma_{0} g_{1}+\sigma_{1} g_{2}\right)+r_{0} r_{1}\left(\sigma_{0} g_{2}+\sigma_{1} g_{3}\right)+r_{1}^{2}\left(\sigma_{0} g_{3}+\sigma_{1} g_{4}\right) \text {. }
$$

Comparing this to eq. (D.2), we see that we have to mod out by the equivalence relations

$$
f_{1} \sim f_{1}+\sigma_{0} g_{1}+\sigma_{1} g_{2}, \quad f_{2} \sim f_{2}+\sigma_{0} g_{2}+\sigma_{1} g_{3}, \quad f_{3} \sim f_{3}+\sigma_{0} g_{3}+\sigma_{1} g_{4} .
$$

Now our aim is to represent $F_{1}$ in matrix form. From (D.9), we see that we can write it as

$$
F_{1}=\left(\begin{array}{cccc}
\sigma_{0} & \sigma_{1} & 0 & 0 \\
0 & \sigma_{0} & \sigma_{1} & 0 \\
0 & 0 & \sigma_{0} & \sigma_{1}
\end{array}\right)
$$

This matrix acts on the column vector $\left(g_{1}, g_{2}, g_{3}, g_{4}\right)^{T}$. Let us now write this matrix in the bases $E_{\alpha}$ in (5.38) and $e_{\beta}$ in (D.7). In this basis, $F_{1}$ is a $7 \cdot 3 \times 2 \cdot 4=21 \times 8$ matrix. To fully express this matrix, we have to study the action of $\sigma_{0}$ and $\sigma_{1}$ on the basis polynomials in (D.7) and present the result in terms of the basis polynomials in (5.38). It is straightforward to show that

$$
\begin{array}{ll}
\sigma_{0} e_{1} \equiv\left(\sigma_{0}\right)_{1}^{\alpha} E_{\alpha}=E_{1}+E_{2}+E_{3}, & \sigma_{0} e_{2} \equiv\left(\sigma_{0}\right)_{2}^{\alpha} E_{\alpha}=E_{4}+E_{5}+E_{6}, \\
\sigma_{1} e_{1} \equiv\left(\sigma_{1}\right)_{1}^{\alpha} E_{\alpha}=E_{4}, & \sigma_{0} e_{2} \equiv\left(\sigma_{0}\right)_{2}^{\alpha} E_{\alpha}=E_{7} .
\end{array}
$$

This leads to the following matrices for $\left(\sigma_{0}\right)_{\beta}^{\alpha}$ and $\left(\sigma_{1}\right)_{\beta}^{\alpha}$ :

$$
\left(\sigma_{0}\right)_{\beta}^{\alpha}=\left(\begin{array}{ll}
1 & 0 \\
1 & 0 \\
1 & 0 \\
0 & 1 \\
0 & 1 \\
0 & 1 \\
0 & 0
\end{array}\right), \quad\left(\sigma_{1}\right)_{\beta}^{\alpha}=\left(\begin{array}{ll}
0 & 0 \\
0 & 0 \\
0 & 0 \\
1 & 0 \\
0 & 0 \\
0 & 0 \\
0 & 1
\end{array}\right) .
$$

Inserting (D.13) into (D.11), gives the full matrix $F_{1}$. 
Performing the quotient action in (D.1) is now equivalent to finding the cokernel of the matrix $F_{1}$ in (D.11), (D.13). This is, in turn, equivalent to finding the kernel of the matrix $\left(F_{1}\right)^{T}$ which acts on the parameters of the polynomials $f_{1}, f_{2}, f_{3}$; that is, on the column vector $\left(a_{1}, \ldots, a_{7}, b_{1}, \ldots, b_{7}, c_{1}, \ldots, c_{7}\right)^{T}$. Finding the kernel of $\left(F_{1}\right)^{T}$ means solving the linear system of equations

$$
\left(F_{1}\right)^{T}\left(a_{1}, \ldots, a_{7}, b_{1}, \ldots, b_{7}, c_{1}, \ldots, c_{7}\right)^{T}=\left(\left(a_{1}, \ldots, a_{7}, b_{1}, \ldots, b_{7}, c_{1}, \ldots, c_{7}\right) F_{1}\right)^{T}=0,
$$

which is equivalent to

$$
\left(a_{1}, \ldots, a_{7}, b_{1}, \ldots, b_{7}, c_{1}, \ldots, c_{7}\right) F_{1}=0
$$

Using the matrix form of $F_{1}$ in eqs. (D.11) and (D.13), it is easy to see that the system of linear equations (D.15) in components becomes (5.40).

Open Access. This article is distributed under the terms of the Creative Commons Attribution License (CC-BY 4.0), which permits any use, distribution and reproduction in any medium, provided the original author(s) and source are credited.

\section{References}

[1] V. Braun, Y.-H. He, B.A. Ovrut and T. Pantev, A heterotic standard model, Phys. Lett. B 618 (2005) 252 [hep-th/0501070] [INSPIRE].

[2] V. Braun, Y.-H. He, B.A. Ovrut and T. Pantev, A standard model from the $E_{8} \times E_{8}$ heterotic superstring, JHEP 06 (2005) 039 [hep-th/0502155] [INSPIRE].

[3] V. Braun, Y.-H. He, B.A. Ovrut and T. Pantev, Vector bundle extensions, sheaf cohomology and the heterotic standard model, Adv. Theor. Math. Phys. 10 (2006) 525 [hep-th/0505041] [INSPIRE].

[4] V. Braun, Y.-H. He, B.A. Ovrut and T. Pantev, The exact MSSM spectrum from string theory, JHEP 05 (2006) 043 [hep-th/0512177] [INSPIRE].

[5] L.B. Anderson, Y.-H. He and A. Lukas, Heterotic compactification, an algorithmic approach, JHEP 07 (2007) 049 [hep-th/0702210] [INSPIRE].

[6] L.B. Anderson, J. Gray, Y.-H. He and A. Lukas, Exploring positive monad bundles and a new heterotic standard model, JHEP 02 (2010) 054 [arXiv:0911.1569] [INSPIRE].

[7] E.I. Buchbinder, A. Constantin and A. Lukas, The moduli space of heterotic line bundle models: a case study for the tetra-quadric, JHEP 03 (2014) 025 [arXiv:1311.1941] [INSPIRE].

[8] E.I. Buchbinder, A. Constantin and A. Lukas, A heterotic standard model with B-L symmetry and a stable proton, JHEP 06 (2014) 100 [arXiv: 1404.2767] [INSPIRE].

[9] E.I. Buchbinder, A. Constantin and A. Lukas, Non-generic couplings in supersymmetric standard models, Phys. Lett. B 748 (2015) 251 [arXiv: 1409.2412] [INSPIRE].

[10] L.B. Anderson, J. Gray, A. Lukas and B. Ovrut, Stabilizing the complex structure in heterotic Calabi-Yau vacua, JHEP 02 (2011) 088 [arXiv: 1010.0255] [INSPIRE]. 
[11] L.B. Anderson, J. Gray, A. Lukas and B. Ovrut, Stabilizing all geometric moduli in heterotic Calabi-Yau vacua, Phys. Rev. D 83 (2011) 106011 [arXiv:1102.0011] [InSPIRE].

[12] M. Dine, N. Seiberg, X.G. Wen and E. Witten, Nonperturbative effects on the string world sheet, Nucl. Phys. B 278 (1986) 769 [INSPIRE].

[13] M. Dine, N. Seiberg, X.G. Wen and E. Witten, Nonperturbative effects on the string world sheet. 2, Nucl. Phys. B 289 (1987) 319 [InSPIRE].

[14] K. Becker, M. Becker and A. Strominger, Five-branes, membranes and nonperturbative string theory, Nucl. Phys. B 456 (1995) 130 [hep-th/9507158] [InSPIRE].

[15] E. Witten, Nonperturbative superpotentials in string theory, Nucl. Phys. B 474 (1996) 343 [hep-th/9604030] [INSPIRE].

[16] R. Donagi, A. Grassi and E. Witten, A nonperturbative superpotential with $E_{8}$ symmetry, Mod. Phys. Lett. A 11 (1996) 2199 [hep-th/9607091] [INSPIRE].

[17] E. Witten, World sheet corrections via D instantons, JHEP 02 (2000) 030 [hep-th/9907041] [INSPIRE].

[18] J.A. Harvey and G.W. Moore, Superpotentials and membrane instantons, hep-th/9907026 [INSPIRE].

[19] E. Lima, B.A. Ovrut, J. Park and R. Reinbacher, Nonperturbative superpotential from membrane instantons in heterotic M-theory, Nucl. Phys. B 614 (2001) 117 [hep-th/0101049] [INSPIRE].

[20] E. Lima, B.A. Ovrut and J. Park, Five-brane superpotentials in heterotic M-theory, Nucl. Phys. B 626 (2002) 113 [hep-th/0102046] [INSPIRE].

[21] E.I. Buchbinder, R. Donagi and B.A. Ovrut, Superpotentials for vector bundle moduli, Nucl. Phys. B 653 (2003) 400 [hep-th/0205190] [INSPIRE].

[22] E.I. Buchbinder, R. Donagi and B.A. Ovrut, Vector bundle moduli superpotentials in heterotic superstrings and M-theory, JHEP 07 (2002) 066 [hep-th/0206203] [INSPIRE].

[23] C. Beasley and E. Witten, Residues and world sheet instantons, JHEP 10 (2003) 065 [hep-th/0304115] [INSPIRE].

[24] C. Beasley and E. Witten, New instanton effects in string theory, JHEP 02 (2006) 060 [hep-th/0512039] [INSPIRE].

[25] C. Schoen, On fiber products of rational elliptic surfaces with section, Math. Z. 197 (1988) 177 .

[26] V. Braun, M. Kreuzer, B.A. Ovrut and E. Scheidegger, Worldsheet instantons, torsion curves and non-perturbative superpotentials, Phys. Lett. B 649 (2007) 334 [hep-th/0703134] [INSPIRE].

[27] V. Braun, M. Kreuzer, B.A. Ovrut and E. Scheidegger, Worldsheet instantons and torsion curves, part A: direct computation, JHEP 10 (2007) 022 [hep-th/0703182] [INSPIRE].

[28] V. Braun, M. Kreuzer, B.A. Ovrut and E. Scheidegger, Worldsheet instantons and torsion curves, part B: mirror symmetry, JHEP 10 (2007) 023 [arXiv:0704.0449] [INSPIRE].

[29] J. Distler, Resurrecting (2,0) compactifications, Phys. Lett. B 188 (1987) 431 [INSPIRE].

[30] J. Distler and B.R. Greene, Aspects of $(2,0)$ string compactifications, Nucl. Phys. B 304 (1988) 1 [INSPIRE]. 
[31] E. Silverstein and E. Witten, Criteria for conformal invariance of $(0,2)$ models, Nucl. Phys. B 444 (1995) 161 [hep-th/9503212] [INSPIRE].

[32] A. Basu and S. Sethi, World sheet stability of $(0,2)$ linear $\sigma$-models, Phys. Rev. D 68 (2003) 025003 [hep-th/0303066] [INSPIRE].

[33] L.B. Anderson, Y.-H. He and A. Lukas, Monad bundles in heterotic string compactifications, JHEP 07 (2008) 104 [arXiv: 0805.2875] [INSPIRE].

[34] R. Bott and L.W. Tu, Differential forms in algebraic topology, Springer, Germany (1982).

[35] P.S. Aspinwall and D.R. Morrison, Chiral rings do not suffice: $N=(2,2)$ theories with nonzero fundamental group, Phys. Lett. B 334 (1994) 79 [hep-th/9406032] [INSPIRE].

[36] B.A. Ovrut, T. Pantev and R. Reinbacher, Torus fibered Calabi-Yau threefolds with nontrivial fundamental group, JHEP 05 (2003) 040 [hep-th/0212221] [INSPIRE].

[37] U. Persson, Configurations of Kodaira fibers on rational elliptic surfaces, Math. Z. 205 (1990) 1.

[38] T. Shioda, "On the Mordell-Weil lattices," Comment. Math. Univ. St.Paul. 39 (1990) 211.

[39] K. Oguiso and T. Shioda, The Mordell-Weil lattice of a rational elliptic surface, Comment. Math. Univ. St.Paul. 40 (1991) 83.

[40] T. Hubsch, Calabi-Yau manifolds: a bestiary for physicists, World Scientific Publishing, Singapore (1992).

[41] P. Candelas, Yukawa couplings between $(2,1)$ forms, Nucl. Phys. B 298 (1988) 458 [INSPIRE].

[42] S. Blesneag, E.I. Buchbinder, P. Candelas and A. Lukas, Holomorphic Yukawa couplings in heterotic string theory, JHEP 01 (2016) 152 [arXiv: 1512.05322] [INSPIRE].

[43] S. Blesneag, E.I. Buchbinder and A. Lukas, Holomorphic Yukawa couplings for complete intersection Calabi-Yau manifolds, arXiv:1607.03461 [INSPIRE].

[44] P. Griffiths and J. Harris, Principles of algebraic geometry, Wiley Classics Library, U.S.A. (2011). 